\title{
ELECTRON-POSITRON INTERACTIONS
}

\section{G. Kramer}

II. Institut für Theoretische Physik der Universität, Hamburg

\section{Introduction}

In these lectures I shall try to summarize some theoretical aspects of $e^{+} e^{-}$interactions ${ }^{1)}$ and try to confront them with recent experimental data from Orsay, Novosibirsk and in particular from Frascati 2). This field of high energy physics has been very interesting in the past, and we can expect further interesting results in the coming years, if we look at table 1, which shows a list of the main electron colliding beam facilities at various laboratories, either completed or under construction.

Table 1

\begin{tabular}{llllcc} 
Laboratory & Ring & Particles & $E_{\max }$ in & $\mathrm{MeV}^{2}$ in $\mathrm{GeV}^{2}$ & Status \\
\hline Orsay & A.C.0. & $\mathrm{e}^{+} \mathrm{e}^{-}$ & 550 & 1.21 & working \\
Novosibirsk & VEPP II & $\mathrm{e}^{+} \mathrm{e}^{-}$ & 700 & 1.96 & $"$ \\
Frascati & Adone & $\mathrm{e}^{+} \mathrm{e}^{-}$ & 1500 & 9.00 & $"$ \\
CEA & Bypass & $\mathrm{e}^{+} \mathrm{e}^{-}$ & 3000 & 36.0 & $"$ \\
SLAC & Spear & $\mathrm{e}^{+} \mathrm{e}^{-}$ & 3000 & 36.0 & $"$ \\
DESY & Doris & $\mathrm{e}^{+} \mathrm{e}^{-}, \mathrm{e}^{-\mathrm{e}^{-}}$ & 3000 & 36.0 & in construction \\
Orsay & D.C.I. & $\mathrm{e}^{+} \mathrm{e}^{-}$ & 1800 & 10.2 & $"$
\end{tabular}

In table 1 the fifth column gives $q^{2}$, the square of the total 
energy in the c.m. system. We see that for the three larger rings at CEA, SLAC and DESY the $q^{2}$ will be comparable to the same quantity achieved at proton accelerators like the Cern PS. Therefore we expect from these rings already results in the asymptotic domain, whereas the other rings, in particular Adone and D.C.I., will work more in the region, where the production of meson and baryon resonances is expected to be dominant.

It is well known that colliding $e^{-e^{-}}$and $e^{+} e^{-}$beams can be used to test quantum electrodynamics at high energies. Since this will be discussed by Conversi in a separate lecture, I shall not consider it here. Another interesting field is the production of strong interacting particles, either by annihilation of electrons and positrons

$$
e^{+} e^{-} \rightarrow \text { hadrons }
$$

or by $e^{ \pm} e^{ \pm}\left(e^{\mp} e^{ \pm}\right)$scattering, as for example

$$
e^{-\bar{\tau}} \rightarrow e^{-e^{\mp}}+\text { hadrons }
$$

Although the processes (1.2) are one order higher in the fine structure constant $\alpha$ than (1.1), there are expectations that the $\gamma \gamma$ processes $(1.2)$ as in Fig. 1 will be observed for higher beam energies $E \geqslant 2 \mathrm{GeV}$, since one factor $\alpha$ in the integrated cross section is partly compensated by a factor $\log \mathrm{E} / \mathrm{me}$, where $w_{e}$ is the electron mass. (1.2) will be of interest in the near 
future in connection with the rings at SLAC and DESY. On the other hand the analysis of processes (1.2) is more complicated than the annihilation process (1.1). Therefore in these lectures we shall concentrate on the annihilation into hadrons and refer for (1.2) to the existing literature ${ }^{3}$ ).

The reactions (1.1) proceed predominantly through one-photon exchange (see Fig. 3). In this case the strong interacting particles $h_{1}, h_{2}, \ldots .$. which appear in the final state,

$$
e^{+} e^{-} \rightarrow h_{1} h_{2} h_{3} \cdot \cdots
$$

must be in a state with parity $P=-1$, charge conjugation quantum number $C=-1$, total angular momentum $J=1$ and total isotopic spin $I=1$ or 0 . If $I=1$, (0) we speak of production by isovector (isoscalar) virtual photons. The final amplitude $\left\langle h_{1}, h_{2}, h_{3}, \ldots\left|g_{\mu}(0)\right| 0\right\rangle$ transforms in the c.m. system of particles $h_{1}, h_{2}, \ldots$ as a polar vector, since, because of the current conservation condition $q^{\mu}\left\langle h_{1}, h_{2}, h_{3}, \ldots\left|g_{\mu}(0)\right| 0\right\rangle=0$ the time component of the current matrix element vanishes $\left\langle h_{1}, h_{2}, h_{3}, \ldots\left|g_{0}(0)\right| 0\right\rangle=0$ and only $\left\langle h_{1}, h_{2}, h_{3}, \ldots|\vec{g}(0)| 0\right\rangle$ is left. of course the one-photon selection rules $P=C=-1, J=1$, $I=0,1$ are valid only to order $\alpha^{2}$ in the intensity and are violated from interference with the two-photon channels at the order $\alpha^{3}$. For production of $n$ pions we must add to these rules the selection rule $I=1$ for $n$ even and $I=0$ for $n$ odd. A unique feature of one-photon annihilation is the form of the angular 
distribution $a+b \cos ^{2} \theta$, if only one particle is detected (i.e. in one-particle inclusive production and two-body annihilation) 1). In the one-photon approximation also the limitation of the cross section by unitarity is rather simple. Since $e^{+} e^{-} \rightarrow h_{1}, h_{2}, \ldots$ proceedes in the direct channel only through total angular momentum $J=1$, it must obey

$$
\sigma_{y} \lesssim \pi \lambda^{2}(2 J+1)
$$

where $\lambda=1 / E$ is the reduced wave length of the incoming $e^{+}$. For $J=1$ and upon taking into account the appropriate spin states of the electrons (1.3) gives

$$
\sigma_{1} \leqslant \frac{3 \pi}{4} \lambda^{2}
$$

The annihilation process takes a particularly simple form in the c.m. system of $e^{+}$and $e^{-}$. Because of the $\gamma_{5}$ - in/variance of the electromagnetic interaction, we have in the limit $\mathrm{m}_{e}=0$ only the coupling of a right-handed electron with a left-handed positron $\left(e_{R}^{-} e_{L}^{+}\right)$and vice versa $\left(e_{L}^{-} e_{R}^{+}\right)$. (See Fig. 2) since the total angular momentum is $J=1$, the only state contributing is

$$
1 / \sqrt{2}\left(\left|e_{L}^{-}, e_{R}^{+}\right\rangle+\left|e_{R}^{-}, e_{L}^{+}\right\rangle\right)
$$


In the past the experimental studies of reactions (1.1) were wostly directed towards the special channels $\pi^{+} \pi^{-}, K \bar{K}$, and $\pi^{+} \pi^{-} \pi^{\circ}$ using the Orsay or Novosibirsk rings ${ }^{2)}$. It is well known that these channels are coupled strongly to the $P=C=-1$ boson resonances $\rho, w$ and $\varphi$, as can be seen from their decay $(\rho \rightarrow 2 \pi, \omega \rightarrow 3 \pi, \varphi \rightarrow \mathrm{kK}, 3 \pi)$. On theoretical grounds it had been expected for a long time that these resonances would couple also to the initial photon 1). This has been verified experimentally in the past years, and we now have a rather detailed knowledge of the electromagnetic coupling of these resonances ${ }^{2)}$. This search for direct resonances in the one-photon channel, which decay into two or more particles, will go on also at higher energies. In this sense the reaction $e^{+} e^{-} \rightarrow$ hadrons is a very unique filter for boson resonances coupled to photons with $\mathrm{P}=\mathrm{C}=-1, \mathrm{~J}=1$ and $I=0,1$.

Since the $\mathrm{e}^{+} \mathrm{e}^{-}$annihilation physics below $2 \mathrm{E}=1 . \mathrm{GeV}$, where these three resonances $\rho, \omega, \varphi$ occur, has been reviewed extensively on other occasions 1)2), I shall concentrate more on the annihilation process in the Frascati energy range $1.0 \mathrm{GeV} \leqslant 2 \mathrm{E} \leqslant 3.0 \mathrm{GeV}$. The plan of the lectures is as follows. In the next section we introduce the total cross section $\sigma$ for $e^{+} e^{-}$annihilation, discuss some simple models, which predict the asymptotic behaviour of $\sigma$, and relate $\sigma$ to the vacuum polarization, which has been measured recently in $e^{+} e^{-} \rightarrow \mu^{+} \mu^{-}$. In section III we explain the kinematics and basic definitions of one-particle distributions and discuss 
their relation to the corresponding process in inelastic electron scattering. The cross section for one-particle detection will be calculated under the assumption of scaling. Section IV gives an introduction to two-particle production for arbitrary spins and parities of the two particles. The general structure of the twoparticle current matrix element is worked out in the c.m. system. This is used to calculate some examples, which later are of importance for the discussion of recent experimental data for many pion production coming from Adone ${ }^{4}$ ). In Section $V$ we give some conclusions and outline the general importance of the study of many-pion channels also at higher energies. 


\section{Total Annihilation Cross Section}

We consider the electron-positron annihilation into an arbitrary final hadron state $H$ (with total momentum $p_{H}$ ). In the onephoton exchange approximation the transition matrix element is written as the product of two matrix elements of the electromagnetic current as indicated by the right half of Fig. 3 . We define $q=k_{+}+k_{-}$. Then the transition matrix element is:

$\left\langle H|T| k_{+}, r_{+} ; k_{-}, r_{-}\right\rangle=-\frac{e^{2}}{q^{2}} \frac{1}{(2 \pi)^{3}} \bar{v}_{r_{+}}\left(k_{+}\right) \gamma^{\mu} u_{r_{-}}\left(k_{-}\right)\langle H|j \mu| 0\rangle$

where $u_{r_{-}}\left(k_{-}\right)$is the Dirac spinor of the electron with momentum $k_{-}$and helicity $r_{-}$and $V_{k_{+}}\left(k_{+}\right)$is the Dirac spinor of the positron with momentum $k_{+}$and helicity $r_{+}$. Then the total cross section is:

$\sigma=\sum_{H} \frac{1}{4} \sum_{r_{+} r_{2}}(2 \pi)^{4} \delta^{(4)}\left(p_{H}-k_{+}-k_{-}\right)\left|\left\langle H|T| k_{+}, r_{+} ; k_{-}, r\right\rangle\right|_{\frac{1}{\phi}}^{2}(2.2)$

Here $\phi$ is the flux factor of the incoming particles

$$
\phi=\frac{4}{(2 \pi)^{6}}\left(\left(k_{+} k_{-}\right)^{2}-m_{e}^{4}\right)^{1 / 2}=\frac{2}{(2 \pi)^{6}}\left(q^{2}\left(q^{2}-4 m_{e}^{2}\right)\right)^{1 / 2}
$$


- $410-$

$w_{e}$ is the electron mass, which can be neglected in Eq. (2.3) for $q^{2} \gg 4 m_{e}^{2}$.. $\sum_{H}$ means integration over the relative momenta of the state $H$, summation over all possible states $H$ with total momentum $p_{H}$ and integration over $p_{H}$. As usual we introduce two tensors, one coming from the lepton vertex, which is for unpolarized electron and positron beams:

$$
\begin{aligned}
t_{\mu \nu} & =\frac{1}{4} \sum_{r_{+}, r} \bar{v}_{r_{+}}\left(k_{+}\right) \gamma_{\mu} u_{r_{-}}\left(k_{-}\right) \bar{u}_{r_{-}}\left(k_{-}\right) \gamma_{\nu} v_{r_{+}}\left(k_{+}\right) \\
& =k_{+\mu} k_{-\nu}+k_{-\mu} k_{+\nu}-9^{2} / 2 g_{\mu \nu}
\end{aligned}
$$

$(2 \cdot 4)$

The other tensor, describing the hadron vertex is:

$$
\begin{aligned}
\prod_{\mu \nu}^{h}(q) & =\sum_{H} \delta^{(4)}\left(p_{H}-q\right)(2 \pi)^{3}\left\langle H\left|j_{\mu}(0)\right| 0\right\rangle\left\langle H\left|g_{v}(0)\right| 0\right\rangle^{*} \\
& =\int d^{4} p_{H} \theta\left(p_{H 0}\right) \delta^{(4)}\left(p_{H}-q\right) \sum_{H}(2 \pi)^{3}\left\langle 0\left|g_{\nu}(0)\right| H\right\rangle\left\langle H\left|j_{\mu}(0)\right| 0\right\rangle \\
& =\theta(q 0) \sum_{H, p_{H}=q}(2 \pi)^{3}\left\langle 0\left|f_{\nu}(0)\right| H\right\rangle\left\langle H\left|g_{\mu}(0)\right| 0\right\rangle
\end{aligned}
$$

With the definition (2.5) we can write the total annihilation cross section as:

$$
\sigma=\frac{\pi e^{4}}{q^{6}} \prod_{\mu \nu}^{h} t^{\mu \nu}
$$


The index $h$ is to indicate that only hadronic states are included in the sum over final states in (2.6) or intermediate states in (2.5). The tensor $\prod_{\mu \nu}^{h}$ depends only on the four vector $q$ as already indicated. Because of the conservation of the electromagnetic current we have:

$$
q^{\mu} \pi_{\mu \nu}^{h}(q)=\sigma
$$

Therefore $\prod_{\mu \nu}^{h}(q)$ depends only on one scalar function $\pi^{h}\left(q^{2}\right)$, defined in the following form

$$
\prod_{\mu \nu}^{h}(q)=\left(q_{\mu} q_{\nu}-g_{\mu \nu} q^{2}\right) \pi^{h}\left(q^{2}\right)
$$

Saturating the leptonic and hadronic tensor we have from:

$$
t^{\mu \nu}\left(q_{\mu} q_{\nu}-g_{\mu \nu} q^{2}\right)=q^{2}\left(q^{2}+2 m_{e}^{4}\right) \approx q^{4}
$$

the result:

$$
\sigma=16 \pi^{3} \alpha^{2} / q^{2} \pi^{h}\left(q^{2}\right)
$$

This way we have expressed the total cross section by one scalar function $\pi^{h}\left(q^{2}\right)$ which depends on one variable $q^{2}=4 \mathrm{k}_{+0}{ }^{2}$ only, where $2 k_{+o}$ is the total energy in the c.m. system $\vec{q}=0$.

The most interesting question is about the asymptotic behaviour 
of $\sigma\left(q^{2}\right)$ for large $q^{2}$. There have been discussed many proposals for this limiting behaviour. We shall consider some of them. If the $q^{2}$ dependence for large $q^{2}$ coincides with that of a pointlike boson it is (see Eq. 4.29 with $F_{\pi}=1$ ):

$$
\sigma \simeq \frac{\pi \alpha^{2}}{3 q^{2}}
$$

and with a pointlike fermion, then (see Eq. (4.42) with $f_{1}=1$, $\left.f_{2}=0\right)$

$$
\sigma \simeq \frac{4 \pi \alpha^{2}}{3 q^{2}}
$$

(2.12) is also the asymptotic value of $\mu^{+} \mu^{-}$production cross section (neglecting radiative corrections). Formula (2.11) and (2.12) are the basis of a the "jet model" of Cabibbo, Parisi and Testa. They assume that the interaction of the virtual photon is mediated by structureless particles (partons). These partons turn themselves into the observed physical particles that retain the total momentum of their primitive unclothed ancestors (see Fig.4a). of course, this presupposes that the number of physical particles originating from any parton is sufficiently small that each final particle carries a significant fraction of the initial parton momentum. Concerning the absolute value of the asymptotic cross section the model predicts that the cross section is equal to (2.11) or (2.12) times the sum of squares of the parton charges, (2.11) if the partons are bosons $(\pi, K, \rho, \ldots)$ and $(2.12)$ if they 
are fermions $(p, \Sigma, \ldots)$. For example, for quarks the asymptotic value is $2 / 3$ times the asymptotic cross section for $\mu$-pair production (2.12). In the parton picture the basic assumption is, that for large $\mathrm{q}^{2}$ the strong interaction, which governs particle interaction in productions by real photons or virtual photons with small $\mathrm{q}^{2}$, is very much reduced, so that the primary interaction with the photon is pointlike. Then there is still the question of the $q^{2}$ dependence of the hadron-hadron interaction, which produces the many hadron final state. Suppose the primary hadron that interacts with the photon is a proton. Then the cross section $\sigma$ for $e^{+} e^{-} \rightarrow$ H can be factorized in one term corresponding to the production of the initial proton-antiproton pair and another one for $p \bar{p} \rightarrow H$ (see Fig. 4b) 6)

$$
\sigma=\frac{4 \pi \alpha^{2}}{3 q^{2}} \quad \frac{q^{2}}{64 \pi} \sigma_{p \bar{p} \rightarrow H}^{J=1}
$$

$$
\begin{aligned}
& \text { Here } \sigma_{p \bar{p} \rightarrow H}^{J=1} \text { is the total cross section for } p \bar{p} \rightarrow \text { H } \\
& \text { with } p \bar{p} \text { in the total angular momentum state } J=1 \text {. If } \sigma=1 \text { is } \\
& \text { described as in a diffractive model for } p \bar{p} \text { interactions with } \\
& \sigma_{\text {tot }} \text { being constant in energy and having the momentum transfer } \\
& \text { dependence } e^{\text {At the partial cross section for arbitrary } J \text { is: }}
\end{aligned}
$$

$$
\text { - } \sigma_{\bar{p} p \rightarrow H}^{y}=\frac{2 \sigma_{\text {tot }}}{q^{2} A} e^{-\tau^{2} / q^{2} A}
$$


The combination of (2.14) with (2.13) gives the result that $\sigma$ behaves like $1 / q^{2}$ for large $q^{2}$, but with reduced magnitude compared to the pointlike cross section. On the other hand we must admit that $e^{A t}$ is presumably a too crude approximation to calculate the $J=1$ partial cross section. For this we need the total angular distribution for $\bar{p} p$ collisions. Therefore the result (2.14) cannot be trusted. Furthermore the primary hadron should rather be a quark than a proton since the latter, we know, is not structureless.

other proposals for the $q^{2}$ dependence of the total $e^{+} e^{-}$cross section are the quark-model estimates of Bjorken'or the one of 8)

Gribov, Ioffe and Pomeranchuk based on the current algebra of free field or recent light-cone derivations, which, concerning the physical assumptions, are similar to the parton model and all result in the $1 / q^{2}$ dependence of $\sigma$.

On the other hand in the vector-dominance model (VDM), in which the sum over intermediate states $H$ is approximated by a finite number of vector meson resonances the conclusion for the total cross sections are different. The predictions range from $\sigma \sim \frac{1}{q}$ for straightforward VDM (finite width corrections may modify this), over $\frac{1}{q^{6} \ln q^{2}}, \frac{1}{q^{4} \ln q^{2}} \quad$ to $\frac{1}{q^{2}}$ depending on the kind of field-theoretic realization of vector dominance. Since this has been extensively reviewed in the literature we shall not discuss it here any further.

The hadronic tensor $\pi_{\mu \nu}^{h}$ as defined in (2.5) appears also 
in pure leptonic reaction as for example $e^{+} e^{-} \rightarrow e^{+} e^{-}$or $e^{+} e^{-} \rightarrow \mu^{+} \mu^{-}$through its contribution to the vacuum polarization. In fact the Fourier transform of the complete Feynman propagator

$$
-\frac{1}{2} D_{\mu \nu}^{F}(x)=\left\langle 0\left|T\left(A_{\mu}(x) A_{\nu}(0)\right)\right| 0\right\rangle
$$

where $A_{\mu}(x)$ is the electromagnetic field, can be written as the sum of the free field propagator and of a gauge invariant correction

$$
-\frac{1}{2} D_{\mu \nu}^{F}(q)=-i\left(g_{\mu \nu} \frac{1}{q^{2}}-\left(g_{\mu \nu}-\frac{q \mu q \nu}{q^{2}}\right) e^{2} \int_{0}^{\infty} d m^{2} \frac{\pi\left(m^{2}\right)}{m^{2}\left(m^{2}-q^{2}-i \varepsilon\right)}\right.
$$

In (2.16) the spectral function $\Pi\left(\mathrm{m}^{2}\right)$ is just the function defined in (2.5) and (2.8), if $\jmath_{\mu}(x)$ is the complete electromagnetic current including the lepton contributions. Using (2.10) we can express the hadronic contribution to the vacuum polarization as an integral over the $e^{+} e^{-}$total cross section. Let us denote the vacuum polarization contribution in $(2.16)$ by $\pi^{F}\left(q^{2}\right)$ Then we get for the hadronic part:

$$
\left(\pi^{F}\left(q^{2}\right)\right)_{\text {hadronic }}=\frac{1}{4 \pi^{2} \alpha} \int_{0}^{\infty} d m^{2} \frac{\sigma\left(m^{2}\right)}{m^{2}-q^{2}-i \varepsilon}
$$


We notice, that in order to obtain a finite value for $\prod_{\text {hadronic }}^{I}$ $\sigma\left(m^{2}\right)$ has to decrease for $m^{2} \rightarrow \infty$ as $\left(\ln m_{n}^{2}\right)^{-2}$ or faster (roughly speaking).

A possible way to detect the hadronic contribution to the vacuum polarization is to measure the process

$$
e^{+} e^{-} \rightarrow \mu^{+} \mu^{-}
$$

The cross section for this process can be factorized in the form

$$
\frac{d \sigma}{d \cos \theta}=\frac{d \sigma_{\text {QED }}}{d \cos \theta}\left|1-q^{2} \pi^{F}\left(q^{2}\right)\right|^{2}
$$

where the point-like cross section $\sigma_{Q E D}$ can be inferred from our calculations of two-body cross sections for spin $1 / 2$ in chapter 3 (see (3.59) with $f_{1}=1, f_{2}=0$ ).

$$
\frac{d \sigma_{Q E D}}{d \cos \theta}=\frac{\pi \alpha^{2}}{2 q^{2}} \frac{2 p}{\sqrt{q^{2}}}\left(\left(1+\cos ^{2} \theta\right)+\frac{4 m_{\mu}^{2}}{q^{2}} \sin ^{2} \theta\right)
$$

Since $\prod^{F}$ is proportional to $\alpha$ the contribution of $\pi^{F}$ in (2.19) will be negligibly small in general. Only if $\sqrt{q^{2}}$ is equal to a resonance mass like that of $\rho$, w or $\varphi$ the term $q^{2} \pi^{F}\left(q^{2}\right)$ may become large enough to be detectable. The experiment has been done recently for $q^{2}$ in the vicinity of $m_{\varphi}^{2}$. The result of the 
measurement as a function beam energy $E$ is seen in Fig. 5. The experimental curve can be fitted by

$$
\sigma=\sigma_{Q E D}\left|1+\frac{3 B}{\alpha} \frac{m_{\varphi} \Gamma}{q^{2}-m_{\varphi}^{2}+i m_{\varphi} \Gamma}\right|^{2}
$$

which is the result of an evaluation of (2.19) for a resonance and $B$ is the branching ratio for $\varphi \rightarrow e^{+} e^{-}$to $\varphi \rightarrow$ all channels and $\mathbf{I}$ is the total width of the $\varphi$. The resulting branching ratio is

$$
B=(2.6 \pm 1.0) \quad 10^{-4}
$$

From this we conclude that the experiment gives evidence for hadronic vacuum polarization.

\section{One-Particle Distributions}

In this section we consider the case where only one particle is detected in the final state, as for example one pion, kaon or 11) proton. The spin of this particle is $S, m$ is its mass and $p$ its energy-momentum four vector. We assume that the polarization of this particle is not detected. The final state will be written as $|\lambda, p, x\rangle$, where $\lambda$ is the helicity of the detected particle 
and $X$ stands for all other particles in the final state. Then the tensor, describing the hadronic vertex is:

$$
\begin{aligned}
& T_{\mu \nu}(p, q)=\sum_{x, \lambda} \delta^{(4)}\left(p_{x}+p-q\right)(2 \pi)^{6}\left\langle\lambda, p_{i} X\left|j_{\mu}(0)\right| 0\right\rangle\left\langle\lambda, p_{j} X\left|\partial_{v}(0)\right| 0\right\rangle^{*} \\
& =\int d M_{x}^{2} \int d^{4} p_{x} \theta\left(p_{x_{0}}\right) \delta\left(p_{x}^{2}-M_{x}^{2}\right) \delta^{(4)}\left(p_{x}+p-q\right)\left(x_{0}^{6}\right)^{6}\left\langle\lambda p_{j} x\left|g_{\mu}(0)\right| 0\right\rangle\left\langle 0\left|j_{v}(0)\right| \lambda, p_{;} x\right\rangle \\
& =\theta\left(q_{0}-p_{0}\right) \sum_{x_{1} \lambda_{i} p_{x}=q-p}(2 \pi)^{6}\left\langle 0\left|g_{v}(0)\right| \lambda, p_{i} x\right\rangle\left\langle\lambda p_{i} x|j \mu(0)| 0\right\rangle
\end{aligned}
$$

The tensor $T_{\mu \nu}$ depends on the two four vectors $p$ and $q$. Furthermore

$$
q^{\mu} T_{\mu \nu}=q^{\nu} T_{\mu \nu}=0
$$

because of current conservation. Then the wost general form for

$$
\begin{aligned}
& T_{\mu \nu} \text { is: } \\
& T_{\mu \nu}=\left(-g_{\mu \nu}+\frac{q_{\mu} q_{\nu}}{q^{2}}\right) W_{1}+\left(p_{\mu}-\frac{p \cdot q}{q^{2}} q_{\mu}\right)\left(p_{\nu}-\frac{p \cdot q}{q^{2}} q_{\nu}\right) \frac{W_{2}}{m^{2}}
\end{aligned}
$$

The two structure functions $w_{1}$ and $w_{2}$, which have been introduced in close analogy to electron scattering, depend only on two scalars for which we choose $\mathrm{q}^{2}$ and the total mass squared of the undetected particles, called $S$ :

$$
S=p_{x}^{2}=(p-q)^{2}=q^{2}+m^{2}-2 p \cdot q=q^{2}+m^{2}-2 \nu
$$


For $p . q$ we shall also use the common symbol $\nu$. If we saturate the leptonic and hadronic tensors we obtain two invariants $I_{1}$ and $I_{2}:$

$$
\begin{aligned}
& I_{1}=-g_{\mu \nu} t^{\mu \nu}=q^{2}+2 m_{e}^{2}=q^{2} \\
& I_{2}=\frac{1}{m^{2}} p_{\mu} p_{\nu} t^{\mu \nu}=\frac{1}{m^{2}}\left\{2 k_{+} \cdot p k_{-} \cdot p-m^{2} q^{2} / 2\right\}
\end{aligned}
$$

Then the differential cross section for inclusive production of a particle of momentum $p$ and spin S becomes:

$$
d \sigma=\frac{\pi e^{4}}{(2 \pi)^{3} q^{6}}\left\{I_{1} W_{1}+I_{2} W_{2}\right\} \frac{d^{3} p}{2 p_{0}}
$$

The invariant differential element $\quad \delta\left(p^{2}-m^{2}\right) \theta\left(p_{0}\right) d^{4} p=\frac{d^{3} p}{2 p_{0}}$ can be evaluated in the c.m. system $\vec{q}=0$, then

$$
\frac{d^{3} p}{2 p_{0}}=\pi|\vec{p}| d p_{0} d \cos \theta
$$

The invariant $I_{2}$ can also be evaluated in this system:

$$
I_{2}=\frac{q^{2} \vec{p}^{2}}{2 m^{2}} \sin ^{2} \theta
$$


Then the cross section $(3.6)$ is:

$$
\frac{d^{2} \sigma}{d p_{0} d \cos \theta}=2 \pi \alpha^{2}|\vec{p}| / q^{4}\left\{\frac{\vec{p}^{2}}{2 m^{2}} \sin ^{2} \theta W_{2}+W_{1}\right\}
$$

The functions $W_{1}$ and $W_{2}$ are free of kinematical singularities and depend on the invariant variables $q^{2}$ and $s$. Instead of the c.m. energy $p_{0}$ and c.m. momentum $|\vec{p}|$ of the observed particle one can use the invariant variable $s$, the invariant mass squared of the unobserved particles. The connection is:

$$
\begin{aligned}
p_{0}=\frac{1}{2 \sqrt{q^{2}}}\left(q^{2}+m^{2}-s\right) & \\
|\vec{p}|=\left(p_{0}^{2}-m^{2}\right)^{1 / 2} & =\frac{1}{2 \sqrt{q^{2}}}\left\{q^{4}+s^{2}+m^{4}-2 q^{2} m^{2}-2 q^{2} s-2 s m^{2}\right\}^{1 / 2} \\
& =\frac{1}{2 \sqrt{q^{2}}} \lambda^{1 / 2}\left(q^{2}, s, m^{2}\right) \\
& =\frac{1}{2 \sqrt{q^{2}}}\left\{\left(s-\left(\sqrt{q^{2}}-m\right)^{2}\right)\left(s-\left(\sqrt{q^{2}}+m\right)^{2}\right)\right\}^{1 / 2} \\
& =\frac{1}{2 \sqrt{q^{2}}}\left\{\left(q^{2}-(\sqrt{s}+m)^{2}\right)\left(q^{2}-(\sqrt{s}-m)^{2}\right)\right\}^{1 / 2}
\end{aligned}
$$

The cross section is:

$$
\frac{d^{2} \sigma}{d s d \cos \theta}=\frac{\pi \alpha^{2}}{q^{4}} \frac{|\vec{p}|}{\sqrt{q^{2}}}\left\{\frac{\vec{p}^{2}}{2 m^{2}} \sin ^{2} \theta W_{2}+W_{1}\right\}
$$


It follows from (2.10), that the variable s varies between the limits :

$$
m^{2} \leqslant s \leqslant\left(m-\sqrt{q^{2}}\right)^{2} \quad ; \quad 4 m^{2} \leqslant q^{2}
$$

The limiting curves, given by (3.12), are shown in Fig. 6, together with the corresponding limits for inelastic electron scattering on pions. Our final result (3.9) or (3.11) shows that the cross section for inclusive one-particle production depends on two structure functions $W_{1}$ and $W_{2}$. The cross section is a linear function of $\sin ^{2} \theta$, characteristic of one-photon exchange. By measuring the distribution in $\sin ^{2} \theta$ both structure functions can be determined.

The diagonal elements of the tensor $T_{\mu \nu}$ are definite positive functions of $\mathrm{q}^{2}$ and $\mathrm{s}$ in the physical region. From this we obtain the following inequalities for the structure functions

$$
\begin{aligned}
& W_{1}\left(q^{2}, s\right) \geqslant 0 \\
& W_{1}\left(q^{2}, s\right)+\frac{\vec{p}^{2}}{\pi^{2}} W_{2}\left(q^{2}, s\right) \geqslant 0
\end{aligned}
$$

where for $\vec{p}^{2}$ we can introduce the invariant expression (3.10) or

$$
\vec{p}^{2}=\left((p q)^{2}-m^{2} q^{2}\right) / q^{2}
$$


The differential cross section can then be written instead of $(3 \cdot 11)$

$\frac{d^{2} \sigma}{d s d \cos \theta}=\frac{\pi \alpha^{2}|\vec{p}|}{2 q^{4} \sqrt{q^{2}}}\left\{\left(1-\cos ^{2} \theta\right)\left(\frac{\vec{p}^{2}}{m^{2}} W_{2}+W_{1}\right)+\left(1+\cos ^{2} \theta\right) W_{1}\right\}$

Then the coefficients of $\left(1-\cos ^{2} \theta\right)$ and $\left(1+\cos ^{2} \theta\right)$ are definite positive functions of $q^{2}$ and $s$.

As the next step we show how $W_{1}$ and $W_{2}$ can be related to decay widths of time-like photons with specified polarization, transverse or longitudinal. This decomposition of $\mathrm{W}_{1}$ and $\mathrm{W}_{2}$ is analogous to the decomposition of the structure functions for inelastic electron scattering into total cross sections for the absorption of transverse or longitudinal space-like photons. The

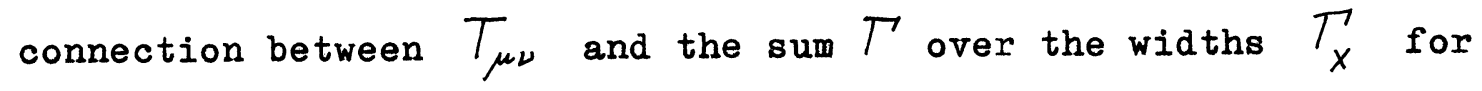
the decay $r \rightarrow p+X$ is

$$
d T_{\mathcal{P}}=\sum_{x} d T_{x \mathcal{P}}=\alpha \frac{|\vec{p}|}{\sqrt{q^{2}}} \epsilon_{\mathcal{P}}^{\mu} \epsilon_{\mathcal{P}}^{\nu *} T_{\mu \nu} d p_{0}
$$

where $\epsilon_{\mathcal{P}}^{\mu}$ is the polarization vector of the time-like photon with polarization $P$. The sum over transverse and longitudinal polarizations $P$ is given by the following projection operator 
- $423-$

$$
\sum_{p= \pm 1} \epsilon_{p}^{\mu} \epsilon_{p}^{\nu *}+\epsilon_{e}^{\mu} \epsilon_{e}^{\nu}=q_{\mu} q_{\nu} / q^{2}-g_{\mu \nu}
$$

With this we find for $2 \mathrm{~d} \Gamma_{t}+\mathrm{d} \Gamma_{\text {from }}(3.16)$ and $(3.3)$

$$
2 \frac{d T_{t}}{d p_{0}}+\frac{d T_{l}}{d p_{0}}=\frac{\alpha|\vec{p}|}{\sqrt{q^{2}}}\left(\frac{\vec{p}^{2}}{m^{2}} W_{l}+3 W_{1}\right)
$$

In the rest system of the virtual photon $\vec{q}=0$ the longitudinal polarization vector $\epsilon_{l}^{\mu}$ has the following form:

$$
\epsilon_{l}^{\mu}=(\sigma,-\vec{p} /|\vec{p}|)
$$

so that

$$
\epsilon_{l}^{\mu} \epsilon_{l}^{\nu *}= \begin{cases}p_{\mu} p_{\nu} / \vec{p}^{2} & ; \mu, \nu=1,2,3 \\ \sigma & \mu \text { or } \nu=0\end{cases}
$$

From this we obtain for d $\Gamma_{\ell}$

$$
\frac{d P_{l}}{d p_{0}}=\frac{\alpha|\vec{p}|}{\sqrt{q^{2}}}\left(W_{1}+\frac{\vec{p}^{2}}{m^{2}} W_{2}\right)
$$


Then $(3.18)$ and $(3.21)$ yield for $d T_{t} / d p_{0}$

$$
\frac{d T_{t}}{d p_{0}}=\frac{\alpha|\vec{p}|}{\sqrt{q^{2}}} W_{1}
$$

and the differential cross section (3.9) or (3.15) takes the form:

$\frac{d^{2} \sigma}{d \cos \theta d p_{0}}=\frac{\pi \alpha}{q^{2} \sqrt{q^{2}}}\left\{\left(1+\cos ^{2} \theta\right) \frac{d T_{t}}{d p_{0}}+\left(1-\cos ^{2} \theta\right) \frac{d T_{e}}{d p_{0}}\right\}$

and after we perform the angular integration:

$$
\frac{d \sigma}{d p_{0}}=\frac{4 \pi \alpha}{3 q^{2} \sqrt{q^{2}}}\left\{2 \frac{d \Gamma_{t}}{d p_{0}}+\frac{d T_{e}}{d p_{0}}\right\}
$$

We see that the combinations (3.13) of the structure functions which are positive definite are just the transverse and longitudinal widths as it should be. The reverse relations are:

$$
\begin{aligned}
& W_{1}=\frac{\sqrt{q^{2}}}{\alpha|\vec{p}|} \frac{d T_{t}}{d p_{0}} \\
& W_{2}=\frac{\sqrt{q^{2}}}{\alpha|\vec{p}|} \frac{m^{2}}{|\vec{p}|^{2}}\left(\frac{d T_{t}}{d p_{0}}-\frac{d T_{l}}{d p_{0}}\right)
\end{aligned}
$$


In the following we shall discuss possible connections with the structure function of inelastic electron scattering, in particular in the kinematic region, where $\mathrm{q}^{2}$ and $s$ are large with

$$
\omega=\frac{2 \nu}{q^{2}}=1+\frac{m^{2}-s}{q^{2}}
$$

kept fixed. Instead of (3.1) and (3.3) the inelastic electron scattering

$$
e^{-} p \rightarrow e^{-} x
$$

where $p$ stands for proton, is described by the tensor $\tilde{T}_{\mu \nu}(p, q)$ where

$\tilde{T}_{\mu \nu}(p, q)=\sum_{x, \lambda} \delta^{(4)}\left(p_{x}-p-q\right)(2 \pi)^{6}\left\langle\lambda, p\left|j_{\mu}(0)\right| x\right\rangle\left\langle x\left|g_{v}(0)\right| p, \lambda\right\rangle$

$\tilde{T}_{\mu \nu}$ has the same decomposition into invariant structure functions $\tilde{W}_{1}\left(q^{2}, s\right) \equiv \tilde{W}_{1}\left(q^{2}, \nu\right)$ and $\tilde{W}_{2}\left(q^{2}, s\right) \equiv \tilde{W}_{2}^{\prime}\left(q^{2}, \nu\right)$ as written down for $T_{\mu \nu}$ in (3.3). The two processes $e^{-p} \rightarrow e^{-x}$ and $e^{+} e^{-} \rightarrow \bar{p} X$ are connected by the substitution law:

$$
T_{\mu \nu}(p, q)=-\tilde{T}_{\mu \nu}(-p, q)
$$

which leads to the following crossing relations for the invariant 
functions :

$$
\begin{aligned}
W_{1}\left(q^{2}, \nu\right) & =-\tilde{W_{1}}\left(q^{2},-\nu\right) \\
\nu W_{2}\left(q^{2}, \nu\right) & =(-\nu) \tilde{W}_{2}\left(q^{2},-\nu\right)
\end{aligned}
$$

where $s$ in $\tilde{W}_{1}$ and $\tilde{W}_{2}$ now is $s=(p+q)^{2}$, but $\nu$ is always $\nu \equiv p \cdot q$ Instead of the variables $q^{2}$ and $s$ we use $q^{2}$ and $w$ and write for spacelike $q^{2}$

$$
\begin{aligned}
\tilde{W}_{1}\left(q^{2}, \nu\right) & \equiv \tilde{F}_{1}(\omega, s) \\
\frac{\nu}{m^{2}} \tilde{W}_{2}\left(q^{2}, \nu\right) & \equiv \tilde{F}_{2}(\omega, s)
\end{aligned}
$$

where $\quad \omega=-\frac{2 \nu}{q^{2}}=1+\frac{m^{2}-s}{q^{2}}>1 \quad$ and $s=(p+q)^{2}=m^{2}+q^{2}+2 \nu$. In the Bjorken Iimit $\left(-q^{2} \rightarrow \infty\right.$, $\nu$ or $s \rightarrow \infty$, w fixed), we have

$$
\begin{aligned}
& \lim _{\nu_{i} q^{2} \rightarrow \infty} \tilde{W_{1}}\left(q^{2}, \nu\right)=\tilde{F_{1}}(\omega)=\lim _{s \rightarrow \infty} \tilde{F}_{1}(\omega, s) \\
& \lim _{\nu_{1}-q^{2} \rightarrow \infty} \frac{\nu}{m^{2}} \tilde{W_{2}}\left(q^{2}, \nu\right)=\tilde{F_{2}}(\omega)=\lim _{s \rightarrow \infty} \tilde{F}_{2}(\omega, s)
\end{aligned}
$$

In the same way we define functions $F_{1}(\omega, s), F_{2}(\omega, s)$ and their limits for $s \rightarrow \infty, q^{2} \rightarrow \infty$ with ${ }^{\omega}$ fixed,,$F_{1}(\omega)$ and $F_{2}(\omega)$ for 
timelike $q^{2}$. The functions $F_{i}(\omega)$ are the "analytic continuations" of the corresponding functions $F_{i}(\omega)$ from $\omega \geqslant 1$ (spacelike $q^{2}$ ) to $0 \leqslant w \leqslant 1$ (timelike $q^{2}$ ). In this context one has to keep in mind, that $F_{i}(\omega, s)$ or $F_{i}(\omega)$ are not analytic if one goes from $q^{2}<0$ to $q^{2}>0$ or from $\omega \geqslant 1$ to $0 \leqslant \omega \leqslant 1$, since for $q^{2}>0$ one has cuts in $q^{2}$ and in the definition of $w_{1}$ and $w_{2}$ there are two factors (see $(3.3)$ and $(3.1))$, namely $\left\langle\lambda, p_{i} \times\left|\jmath_{\mu}(0)\right| 0\right\rangle$ and $\left\langle\lambda, p, X\left|g_{v}(0)\right| 0\right\rangle^{*}$, so that the second factor is to be taken below the cut if the first factor is taken above the cut. only if these cuts in $\mathrm{q}^{2}$ are unimportant, as it is obviously the case in parton models, we can perform the analytic continuation without knowledge about the details of the $q^{2}$ cuts. The data on electron-proton scattering from SLAC and DESY show that $\tilde{W}_{1}$ and $\frac{\nu}{m^{2}} \tilde{W}_{2}$ scale, that means that $F_{1}$ and $F_{2}$ are independent of $s$ and seem to approach neither zero nor infinity for $q^{2}$ or $s$ large. Although if one assumes that Bjorkens original scaling hypothesis for ep scattering is supported by experiment, it is not obvious, that the corresponding structure function in the annihilation process should scale also. As was explained above, reference to the substitution law is not sufficient for this purpose. Actually there are models which show scaling in the spacelike region but have nonscaling structure functions for the annihilation process. 32 )

Nevertheless let us assume that scaling for $q^{2}>0$ is true also, then we get for the cross section for $q^{2}, s \rightarrow \infty$, w fixed: 


$$
\frac{d^{2} \sigma}{d \cos \theta d \omega}=\frac{\pi \alpha^{2}}{2 q^{2}} \omega\left\{F_{1}(\omega)+\frac{\omega}{4} F_{2}(\omega) \sin ^{2} \theta\right\}
$$

In the case of scaling the one-particle inclusive cross section decreases as $1 / q^{2}$ for large $q^{2}$. In the rest system of the photon the variable $\omega$ is

$$
\omega=\frac{2 \nu}{q^{2}}=\frac{p_{0}}{w / 2}
$$

By measuring the cross section for a series of beam energies $W / 2$ and varying proton energies $p_{0}$ so that $\omega$ is fixed the scaling property of $\mathrm{q}^{2} d^{2} \sigma / d \cos \theta d \omega$ could be tested. Since this cross section decreases much less with $q^{2}$ as for example $\bar{p} p$ production this test should be feasable.

We mention that we have

$$
\omega F_{2} / 2+F_{1}=0
$$

if the longitudinal part $d \Gamma_{l} / d p_{0}$ of the cross section should be negligihle compared to the transversal part as it seems to be the case in the spacelike region. Then $F_{2}$ must be negative according to (3.33). The integrated inclusive cross section has the simple form: 


$$
\frac{d \sigma}{d \omega}=\frac{\pi \alpha^{2}}{q^{2}} \omega^{2}\left(-F_{2}(\omega)\right)=\frac{2 \pi \alpha^{2}}{q^{2}} \omega F_{1}(\omega)
$$

\section{Two-Particle Production.}

\section{A. General Form of Cross Section}

In this chapter we shall consider as final hadron state H a two-particle state $\left|s_{a}, \lambda_{a}, s_{b}, \lambda_{b}\right\rangle$, where $s_{i}, \lambda_{i}$ are spin and helicity of particle $i$ respectively. Later we shall work out special examples, like $\pi^{+} \pi^{-}, \pi^{0} \omega, \pi A_{1}, p \bar{p}$ and others and develop a special model based on $\rho$ dominance which will be compared with available data. 12)

We define the current matrix element of the two-hadron state by the symbol

$$
J_{\mu}=(2 \pi)^{3}\left\langle p_{a}, s_{a}, \lambda_{a} ; p_{b}, s_{b}, \lambda_{b}\left|j_{\mu}(0)\right| 0\right\rangle
$$

with labels of particles as in Fig. 7 . The cross section for production of the state $a, b$ by one-photon exchange is expressed by (4.1) and the tensor $t_{\mu \nu}$ of the electron vertex given by (2.4). Then the differential cross section, with $\Omega$ being the 
- $430-$

solid angle of particle a, comes out to be:

$$
\frac{d \sigma}{d \Omega}=\frac{\alpha^{2} p}{2 W^{7}} \sum_{\text {Spins }} t^{\mu \nu} J_{\mu}{J_{\nu}^{*}}^{*}
$$

with $\quad t_{\mu \nu}=k_{+\mu} k_{-\nu}+k_{-\mu} k_{+\nu}-\frac{W^{2}}{2} g_{\mu \nu}$

$p$ is equal to the momentum in the virtual photon rest system $(\vec{q}=0)$, where $q=p_{a}+p_{b}$ and $w=\left(q^{2}\right)^{1 / 2}$, so that

$$
p^{2}=\frac{1}{4 W^{2}}\left(W^{2}-\left(m_{a}+m_{b}\right)^{2}\right)\left(W^{2}-\left(m_{a}-m_{b}\right)^{2}\right)
$$

In this system $(\vec{q}=0)$ and with $\vec{k}_{+}$along the z-direction the tensor $t_{\mu \nu}$ reduces to ( in the $m_{e}=\sigma$ limit):

$$
t_{\mu \nu}=\frac{w^{2}}{2} L_{\mu \nu} \quad \text { with } \quad L_{\mu \nu}=\left\{\begin{array}{l}
\delta_{i j}-\delta_{i 3} \delta_{j 3} \\
\sigma \text { if } \mu \text { or } \nu=0
\end{array}\right.
$$

so that

$$
\frac{d \sigma}{d \Omega}=\frac{\alpha^{2} p}{4 W^{5}} \sum_{\text {spins }} \sum_{m= \pm 1}\left|J_{m}\right|^{2}
$$


where $m= \pm 1$ are the spherical components of $J_{\mu}$. That $d \sigma / d \Omega$ does not depend on $J_{0}$ and $J_{3}$ in obvious, since in the sum

$$
j_{e}^{\mu} J_{\mu}=\mathcal{J}_{e} \circ J_{0}-\vec{J}_{e} \cdot \vec{J}
$$

where $\gamma_{e}^{\mu}$ is the electron current, because of current conservation $q_{0} j_{e}=\vec{q} \cdot \vec{j}_{e}$, the cowponent $j_{e}$ vanishes (of course also $\mathrm{J}_{0}=0$ ) in the photon rest system, whereas $\mathrm{J}_{3}$ drops out because $f_{e}$ vanishes in the limit $m_{e}=0$.

The next step is to give the most general form of $\mathrm{J}_{m}$ in terms of helicity matrix element of total angular momentum (which is equal to one of course) and angel dependent factors. This is achieved, as usual, by expaning the helicity state $\left|p_{a}, s_{a}, \lambda_{a} ; p_{b}, s_{b}, \lambda_{b}\right\rangle$ $\equiv\left|p \theta \phi_{j} \lambda_{a}, \lambda_{b}\right\rangle$ in the c.m. system of particle $a$ and into spherical states.

$$
\begin{aligned}
& \left|p \theta \phi ; \lambda_{a}, \lambda_{b}\right\rangle=\sum_{J, M} N_{J} D_{M \lambda}^{J}(\phi, \theta,-\phi)\left|J, M, \lambda_{a}, \lambda_{b}\right\rangle^{(4.7)} \\
& \text { with } \lambda=\lambda_{a}-\lambda_{b}, N_{J}=((2 J+1) / 4 \pi)^{1 / 2} \quad \text {. If we substitute } \\
& (4.7) \text { into (4.1) we obtain }
\end{aligned}
$$

$$
\begin{gathered}
J_{m}=(2 \pi)^{3}\left\langle p \theta \phi, \lambda_{a}, \lambda_{b}\left|g_{m}(0)\right| 0\right\rangle=\sum_{J, M} N_{y} D_{M \lambda}^{I *}(\phi, \theta,-\phi) \\
\times(2 \pi)^{3}\left\langle I M \lambda_{a} \lambda_{b}\left|g_{m}(0)\right| 0\right\rangle
\end{gathered}
$$


$-432-$

The state $f_{m}(0)|0\rangle$ transforms as a state with total angular momentum $\mathrm{J}=1$ and $z$-component $\mathrm{J}=\mathrm{m}$. Therefore

$$
\left\langle J M \lambda_{a} \lambda_{b}\left|g_{m}(0)\right| 0\right\rangle=\delta_{J 1} \delta_{M m}\left\langle 1 m \lambda_{a} \lambda_{b}\left|g_{m}(0)\right| 0\right\rangle
$$

We define:

$$
(2 \pi)^{3}\left\langle 1, m, \lambda_{a}, \lambda_{b}\left|f_{m}(0)\right| 0\right\rangle \sqrt{\frac{3}{4 \pi}}=\Gamma_{m}^{\lambda_{a} \lambda_{b}}
$$

so that

$$
I_{m}=D_{m \lambda}^{1}(\phi, \theta,-\phi) \Gamma_{m}^{\lambda_{a} \lambda_{b}}
$$

The reduced matrix element $\Gamma_{m}^{\lambda_{a} \lambda_{b}}$, which we shall call the helicity form factor depends only on $q^{2} \equiv w^{2}$. It will have kinemetical singularities and/or zeros which will be determined later.

With (4.11) and

$$
D_{m \lambda}^{1}(\phi, \theta,-\phi)=e^{i(\lambda-m) \phi} d_{m \lambda}^{1}(\theta)
$$

the cross section ( 4.5$)$ becomes:

$$
\frac{d \sigma}{d \Omega}=\frac{\alpha^{2} p}{4 W^{5}} \sum_{\lambda_{a}, \lambda_{b}} \sum_{m= \pm 1}\left(d_{m \lambda}^{1}(\theta)\right)^{2}\left|\Gamma_{m}^{\lambda_{a} \lambda_{b}}\right|^{2}
$$




\section{B. Properties and Number of Independent Form Factors}

Next we shall look at the properties of $T_{m}^{\lambda_{a} \lambda_{b}}$, following from the assumption of rotational invariance and space and time reflection invariance. First we observe that the current operator $f_{m}(0),(m=0, \pm 1)$ transforms under ordinary rotations as an irreducible vector operator. Then we can apply the Wigner-EckartTheorem which states that $(4.10)$ can be written as:

$$
\begin{aligned}
\Gamma_{m}^{\lambda_{a}, \lambda_{b}}=(2 \pi)^{3}\langle 1 m & \left.\lambda_{a} \lambda_{b}\left|j_{m}(0)\right| 0\right\rangle \\
= & \varphi(011 ; 0 \mathrm{~m})(2 \pi)^{3}\left\langle 1 \lambda_{a} \lambda_{b}\left\|y_{m}(0)\right\| 0\right\rangle
\end{aligned}
$$

Since $\ell(011 ; 0 \mathrm{~m})=1$ and the reduced matrix element is independent of the projection quantum number $m, \quad \Gamma_{m}^{\lambda_{a} \lambda_{b}}$ does not depend on $m$. In the following we shall omit this index.

From space reflection $(\nabla)$ invariance, for which we have $P y_{m}(0) P^{-1}=-J_{m}(0)$ and $P\left|I_{1}, \lambda_{a}, \lambda_{b}\right\rangle=$

$$
\begin{gathered}
\eta_{a} \eta_{b}(-)^{I-S_{a}-S_{b}}\left|J_{1} M,-\lambda_{a},-\lambda_{b}\right\rangle \text { we deduce the relation: } \\
\Gamma^{\lambda_{a}, \lambda_{b}}=\eta_{a} \eta_{b} \Gamma^{-\lambda_{a}, \lambda_{b}}
\end{gathered}
$$

where $\eta_{a}=\eta_{a}(-)^{S_{a}}$ is the normality of particle $a$ and $\eta_{a}$ its parity. From time reversal (T) invariance, for which

$$
\begin{aligned}
T_{f_{m}(0) T^{-1}=f_{m}(m= \pm 1) \text { and }} T\left|\mathcal{J}, M, \lambda_{a}, \lambda_{b}\right\rangle \\
=(-)^{\top-M}\left|\mathcal{J},-M, \lambda_{a}, \lambda_{b}\right\rangle
\end{aligned}
$$


we deduce that

$$
\left(\Gamma^{\lambda_{a}, \lambda_{b}}\left(q^{2}-i \varepsilon\right)\right)^{*}=\Gamma^{\lambda_{a}, \lambda_{b}}\left(q^{2}+i \varepsilon\right)
$$

The relation (4.15) states that the helicity form factors are real in the region $q^{2} \leqslant 0$, i.e. for electron scattering, where $q^{2}$ is spacelike.

If particle $a$ and $b$ are charge-conjugate to each other we apply the charge conjugation operator $c$. Since $\varphi\left|I, M, \lambda_{a}, \lambda_{b}\right\rangle=$ $(-)^{I-\lambda_{a}+\lambda_{b}}\left|I_{1}, \lambda_{b}, \lambda_{a}\right\rangle \quad$ and $\quad \varphi f_{\mu}(0) C^{-1}=-f_{\mu}(0)$

we get

$$
\Gamma^{\lambda_{a} \lambda_{b}}=(-)^{\lambda_{b}-\lambda_{a}} \Gamma^{\lambda_{b} \lambda_{a}}
$$

On the basis of $(4.13)$ and the relations (4.14), (4.16) for charge conjugate particles and the restriction that $\left|\lambda_{a}\right| \leqslant S_{a},\left|\lambda_{b}\right| \leqslant S_{b}$ and $|\lambda|=\left|\lambda_{a}-\lambda_{b}\right| \leqslant 1$ we can count the number of independent complex form factors for the three cases $n_{a} n_{b}=1$, $n_{a} n_{b}=-1$ and $a, b$ charge conjugate (called identical particles). The result for bosons is shown in Table 2 and for fermions in Table 3 . 
Table 2: Number of independent form factors for bosons

\begin{tabular}{lllll} 
spin & $n_{a} n_{b}$ & $\lambda=0$ & $\lambda=+1$ & total \\
\hline$s_{a}<s_{b}+$ & $s_{a}+1$ & $2 s_{a}+1$ & $3 s_{a}+2$ \\
\hline$s_{a}=s_{b}+$ & $s_{a}+1$ & $2 s_{a}$ & $3 s_{a}+1$ \\
\hline$s_{a}<s_{b}-$ & $s_{a}$ & $2 s_{a}+1$ & $3 s_{a}+1$ \\
\hline$s_{a}=s_{b}-$ & $s_{a}$ & $2 s_{a}$ & $3 s_{a}$ \\
\hline identical + & $s+1$ & $s$ & $2 s+1$ \\
\hline
\end{tabular}

Table 3: Number of independent form factors for fermions

\begin{tabular}{llll} 
spin & $\lambda=0$ & $\lambda=+1$ & total \\
\hline$S_{a}<S_{b}+$ & $S_{a}+1 / 2$ & $2 S_{a}+1$ & $3 S_{a}+3 / 2$ \\
\hline$S_{a}=S_{b}+$ & $S_{a}+1 / 2$ & $2 S_{a}$ & $3 S_{a}+1 / 2$ \\
\hline$S_{a}<S_{b}-$ & $S_{a}+1 / 2$ & $2 S_{a}+1$ & $3 S_{a}+3 / 2$ \\
\hline$S_{a}=S_{b}-$ & $S_{a}+1 / 2$ & $2 S_{a}$ & $3 S_{a}+1 / 2$ \\
\hline identical - & $S+1 / 2$ & $S+1 / 2$ & $2 S+1$ \\
\hline
\end{tabular}

As some examples let us consider the system $\pi R_{n}$, where $R_{n}$ is a meson resonance with normality $n$. Then $S_{a}=0$ and $n_{a}=-1$. Therefore we have two independent form factors if $n=-1$; examples for $R_{-1}$ are: $A_{1}$ and the $A_{3}$ resonance. If $n=+1$, like the $\rho, w, \varphi$ or $A_{2}$ resonance, we have only one form factor. 
If the particle a is a $0^{+}$resonance instead of $0^{-}$the role of $\mathrm{n}=+1$ resonances and $\mathrm{n}=-1$ resonances is interchanged. Table 3 shows that we need two form factors for the system $p \bar{p}$, three for $\Delta \bar{p}$ and four for the system $\Delta \bar{\Delta}$, where $\Delta$ is for example the well known $3 / 2^{+}$resonance with mass $1.232 \mathrm{GeV}$.

\section{Differential and Total Cross Section for Two-Particle}

\section{Production}

The formula for the differential cross section (4.12) can now be simplified further. Since $\lambda=0, \pm 1$ only we can write for $(4.12)$ the following expression:

$$
\begin{aligned}
\frac{d \sigma}{d \Omega}=\frac{\alpha^{2} p}{4 W^{5}} \sum_{\lambda_{a}} & \left\{\sin ^{2} \theta\left|\Gamma^{\lambda_{a}, \lambda_{a}}\right|^{2}\right. \\
& \left.+\frac{1}{2}\left(1+\cos ^{2} \theta\right)\left(\left|\Gamma^{\lambda_{a}, \lambda_{a}+1}\right|^{2}+\left|\Gamma^{\lambda_{a}, \lambda_{a}-1}\right|^{2}\right)\right\}(4.17)
\end{aligned}
$$

Here we used the explicit formulas for the $d$-functions:

$$
d_{ \pm 1, \lambda}^{1}=\frac{1}{2}(1 \pm \lambda \cos \theta), \quad d_{ \pm 1,0}^{1}=\mp \frac{1}{\sqrt{2}} \sin \theta, \lambda= \pm 1 .
$$

(4.17) is our final formula for the differential cross section.

$\theta$ is the angle between the direction $\vec{k}_{+}$and the momentum $\vec{p}_{a}$ $(4.17)$ is completely general. If we compare it with $(3.23)$, the cross section of inclusive production, we see that the term proportional to $\sin ^{2} \theta$ is the longitudinal cross section and the 
term proportional to $\left(1+\cos ^{2} \theta\right)$ is the cross section for transverse photon polarization:

$$
\begin{aligned}
\frac{d \sigma}{d \Omega} & =\frac{3}{8 \pi}\left(\sin ^{2} \theta \sigma_{l}+\frac{1}{2}\left(1+\cos ^{2} \theta\right) \sigma_{t}\right) \\
\sigma_{l} & =\frac{8 \pi \alpha^{2} p}{1} \sum_{W^{5}} \sum_{\lambda_{a}}\left|\Gamma^{\lambda_{a} \lambda_{a}}\right|^{2} \\
\sigma_{t} & =\frac{8 \pi \alpha^{2} p}{12 W^{5}} \quad\left(\left|\Gamma^{\lambda_{a}, \lambda_{a}+1}\right|^{2}+\left|\Gamma^{\lambda_{a}, \lambda_{a}-1}\right|^{2}\right)
\end{aligned}
$$

The integrated cross section is

$$
\begin{aligned}
& \sigma=\sigma_{e}+\sigma_{t} \\
& =\frac{2 \pi \alpha^{2} p}{3 W^{5}} \sum_{\lambda_{a}}\left\{\left|\Gamma^{\lambda_{a}, \lambda_{a}}\right|^{2}+\right. \\
& \left.\left|\Gamma^{\lambda a_{1} \lambda_{a}+1}\right|^{2}+\left|\Gamma^{\lambda_{a}, \lambda_{a}-1}\right|^{2}\right\}
\end{aligned}
$$

As was to be expected, by measuring the angular distribution of the emitted particles one determines only two functions out of the maximal $3 S_{a}+2,\left(3 S_{a}+3 / 2\right)$ complex form factors. In particular there appears no interference term between longitudinal and transverse photon polarization in $d \sigma / d \Omega$ 
To explain the separation (4.18) directly we can use the definition of longitudinal polarization (3.19) and multiply it with (4.11). Then we obtain:

$$
(2 \pi)^{3}\left\langle p \theta \phi, \lambda_{a}, \lambda_{b}|\vec{j}(0)| 0\right\rangle \vec{\epsilon}_{l}=-\Gamma^{\lambda_{a}, \lambda_{a}}
$$

A similar relation is found between the projection with the transverse polarization vectors and $\Gamma^{\lambda_{a}, \lambda_{a} \pm 1}$.

It is also instructive to write the hadronic tensor in terms of two invariant function $M_{1}$ and $M_{2}$ which depend only on $q^{2} \equiv w^{2}$.

$$
\sum_{\text {spins }} J_{\mu} J_{\nu}^{*}=\left(\frac{q_{\mu} q_{\nu}}{q^{2}}-g_{\mu \nu}\right) M_{1}+\left(p_{\mu}-\frac{p \cdot q}{q^{2}} q_{\mu}\right)\left(p_{\nu}-\frac{P \cdot q_{q}}{q^{2}}\right) M_{2}
$$

where $p=p_{a}-p_{b}, q=p_{a}+p_{b}$. Then

$$
\frac{d \sigma}{d \Omega}=\frac{\alpha^{2} p}{2 W^{5}}\left(M_{1}+\frac{p^{2}}{2 m_{a}^{2}} M_{2} \sin ^{2} \theta\right)
$$

In terms of the single particle distributions $W_{1}$ and $W_{2}$ introduced in $(3,3)$ we have the following relation

$$
\begin{aligned}
& W_{1}=\delta\left(s-m_{b}^{2}\right) M_{1} \\
& W_{2}=\delta\left(s-m_{b}^{2}\right) M_{2}
\end{aligned}
$$


where $s=(p-q)^{2}$ is the missing mass. It is clear that for two particle production the inclusive structure functions $W_{1}$ and $W_{2}$ have a delta distribution in the missing mass

$$
\delta\left(s-m_{b}^{2}\right)=\frac{1}{2 W} \delta\left(p_{0}-\frac{W}{2}\right)
$$

If the particle is unstable the $\delta$-distribution (4.24) is replaced by a Breit-Wigner function.

The coefficients are just the function $\mathrm{M}_{1}$ and $\mathrm{M}_{2}$. They can be expressed by the form factors. The relations are:

$$
\begin{aligned}
& M_{1}=\frac{1}{2} \sum_{\lambda_{a}}\left\{\left|\Gamma_{a_{1}, \lambda_{a}+1}\right|^{2}+\left|\Gamma^{\lambda_{a}, \lambda_{a}-1}\right|^{2}\right\} \\
& M_{2}=\frac{m_{a}^{2}}{p^{2}}\left\{\sum_{a}\left|\Gamma \lambda_{a}, \lambda_{a}\right|^{2}-M_{1}\right\}
\end{aligned}
$$

\section{Relations between Helicity Form Factors and Invariant}

\section{Form Factors}

The helicity form factor $\Gamma^{\lambda_{a} \lambda_{b}}$ contain kinematical singularities and zeros. In the literature one can find general methods to extract these singularities. Since we are interested only in some special examples we shall not make use of these general decompositions of the current matrix elements into standard covariants and invariant form factors free of kinematical 
singularities. Instead we use the standard definitions of invariant form factors for the special cases we are interested in.

The simplest example is the $\pi^{+} \pi^{-}$production by $\mathrm{e}^{+} \mathrm{e}^{-}$annihilation. The current matrix element depends only on one form factor $F_{\pi}$ which is a function of $\mathrm{q}^{2}$ only and free of kinematical singularities. It is related to the current matrix element by:

$$
(2 \pi)^{3}\left\langle\pi^{+}\left(p_{a}\right), \pi^{-}\left(p_{b}\right)\left|j_{\mu}(0)\right| 0\right\rangle=\left(p_{a}-p_{b}\right) \mu F_{\pi}\left(q^{2}\right)
$$

Comparison of $(4.26)$ in the system $\vec{q}=0$ and $(4.11)$ leads to

$$
\Gamma^{\infty 0}=2 p F_{\pi}
$$

We see that the helicity form factor has the characteristic threshold singularity factor $p=\frac{1}{2}\left(q^{2}-4 m_{\pi}^{2}\right)^{1 / 2}$ for a $p$-wave. With (4.27) we obtain for the cross section from (4.17)

$$
\frac{d \sigma}{d \cos \theta}=\frac{2 \pi \alpha^{2}}{W^{2}}\left(\frac{p}{W}\right)^{3}\left|F_{\pi}\right|^{2} \sin ^{2} \theta
$$

and for the integrated cross section:

$$
\sigma=\frac{\pi \alpha^{2}}{3 W^{2}}\left(\frac{2 p}{W}\right)^{3}\left|F_{\pi}\right|^{2}
$$


As to be expected the cross section is purely longitudinal and has the $\sin ^{2} \theta$ dependence.

Next we discuss the final state $\pi^{\circ} \omega$. Of course, the same formulas apply for $\pi^{\circ} \varphi$ and $\pi^{\circ} \rho^{0}$. The covariant current matrix element, which obeys explicitly current conservation, is:

$(2 \pi)^{3}\left\langle\pi^{0}(p a), \omega\left(\lambda_{b}, p_{b}\right)\left|j_{\mu}(0)\right| 0\right\rangle=i F_{\pi \omega \gamma} \epsilon_{\mu \alpha \beta \gamma} p_{a}^{\alpha} q^{\beta} \epsilon^{\lambda_{b}}\left(p_{b}\right)^{\gamma *}(4.30)$

Here $F_{\pi \omega \gamma}$ is the invariant form factor, free of kinematic singularities, and $\epsilon^{\lambda_{b}}\left(p_{b}\right)$ is the polarization vector of the w meson. From (4.30) we have calculated the $\Gamma^{0, \lambda_{b}}$.

$$
\Gamma^{0,0}=\sigma \quad, \quad T^{0, \lambda_{b}}=\lambda_{b} W p F_{\pi \omega \gamma}\left(W^{2}\right)
$$

$\Gamma^{0,0}=0$ follows already from $(4 \cdot 14)$, since $n_{\pi} n_{\omega}=-1$. $\Gamma^{0, \lambda_{b}}$ has kinematical singularities from the factor $W \cdot p$, which produces square-root singularities at the threshold of production $q^{2}=\left(m_{\pi}+m_{\omega}\right)^{2}$ and at the pseudothreshold $q^{2} \equiv W^{2}=\left(m_{\pi}-m_{\omega}\right)^{2}$. The final state $\pi \omega$ is produced only by transversely polarized virtual photons. Therefore the angular distribution is of the form $\left(1+\cos ^{2} \theta\right)$. The complete formulae for the cross sections are: 
$-442-$

$$
\frac{d \sigma}{d \cos \theta}=\pi \alpha^{2}\left(\frac{p}{W}\right)^{3}\left|F_{\pi \omega \gamma}\right|^{2} \frac{1}{2}\left(1+\cos ^{2} \theta\right)
$$

and

$$
\sigma=\frac{\pi \alpha^{2}}{6}\left(\frac{2 p}{W}\right)^{3}\left|F_{\pi \omega \gamma}\right|^{2}
$$

This result for the $\pi \omega$ system can be generalized very easily to a system $\pi R_{S}$, where $R_{S}$ is a boson resonance with spin $s$ and positive normality $n_{S}=\eta(-)^{s}=1$. As an example let us consides the system $\pi \mathrm{A}_{2}$. The $\mathrm{A}_{2}$ meson has spin 2. Instead of (4.30) the current matrix element is in this case:

$$
\begin{aligned}
& (2 \pi)^{3}\left\langle\pi\left(p_{a}\right), A_{2}\left(\lambda_{b}, p_{b}\right)\left|j_{\mu}(0)\right| 0\right\rangle \\
& =i F_{\pi A_{2} \gamma} \epsilon_{\mu \alpha \beta \gamma_{1}} p_{a}^{\alpha} q^{\beta} \epsilon^{\lambda_{b}^{*}}\left(p_{b}\right)^{\gamma_{1} \gamma_{2}} p_{a} \gamma_{2}
\end{aligned}
$$

$\epsilon^{\lambda_{b}}\left(p_{b}\right)^{\gamma_{1} \gamma_{2}}$ is the polarization vector of the $A_{2}$ resonance. It can be calculated from the polarization vector for spin 1 with the help of the following relation:

$$
\epsilon^{\lambda_{b}}\left(p_{b}\right)_{\gamma_{1} \gamma_{2}}=\sum_{\lambda_{1} \lambda_{b}^{\prime}}^{\prime} e\left(112 ; \lambda_{b}^{\prime} \lambda\right) \epsilon^{\lambda_{b}^{\prime}}\left(p_{b}\right)_{\gamma_{1}} \epsilon^{\lambda_{b}}\left(p_{b}\right)_{\gamma_{2}}
$$


Since in the photon rest system:

$$
\epsilon^{\lambda_{b}}\left(p_{b}\right) \cdot p_{a}=\epsilon^{\lambda_{b}}\left(p_{b}\right) \cdot q=-\frac{1}{m_{A_{2}}} p q_{0} \delta_{\lambda_{0}}=-\delta_{\lambda_{0}} \frac{p W}{m_{A_{2}}}
$$

we get the following result for $\Gamma^{0, \lambda_{b}}$ for the $\pi A_{2}$ system with the help of (4.34) (4.35) and the corresponding formula for the $\pi \omega$ system:

$$
\Gamma^{0,0}=\theta \quad, \Gamma^{0, \lambda_{b}}=-\lambda_{b} \frac{(p W)^{2}}{\sqrt{2} m_{A_{l}}} F_{\pi A_{2} \gamma}
$$

so that the cross section is:

$$
\frac{d \sigma}{d \cos \theta}=\frac{\pi x^{2} p^{5}}{2 W m_{A_{2}}^{2}}\left|F_{\pi A_{2} \gamma}\right|^{2} \frac{1}{2}\left(1+\cos ^{2} \theta\right)
$$

It follows clearly from the recurrence relation (4.34) and from (4.35) that the power of the threshold factor increases with the spin of the resonance $R_{S}$ like $(p W)^{s^{\prime}}$ in the matrix element $\Gamma^{0, \lambda_{b}}$ and therefore as $(p \cdot W)^{2 s}$ in the cross section. This with $q^{2}$ increasing factor must be damped by the form factor $F_{\pi R \gamma}\left(q^{2}\right)$. In order to be in accord with unitarity, which means that 
$d \sigma / d \Omega \lesssim 1 / q^{2}$ for $q^{2} \rightarrow \infty$ the form factor $F_{\pi R \gamma}$ has to decrease with $q^{2}$ the more the higher the spin $s$ is. For $\pi \mathrm{A}_{2}$ the form factor $F_{\pi A_{2} \gamma}$ has to decrease at least as $\frac{1}{w^{3}}$ for $W \equiv\left(q^{2}\right)^{1 / 2} \rightarrow \infty$, in order not to violate the unitarity limit for $\sigma$.

As last example for final two-meson states we shall consider the system $\pi \mathrm{A}_{1}$. According to Table 2 the transition current to this final state with $n_{a} n_{b}=1$ is determined by two independent form factors. We denote these two invariant form factors by $F_{\pi A_{1} \gamma}$ and $F_{\pi A_{1} \gamma}^{2}$. They appear in the decomposition of the covariant current matrix element in the following form:

$$
\begin{aligned}
& (2 \pi)^{3}\left\langle\pi\left(p_{a}\right), A_{1}\left(\lambda_{b}, p_{b}\right)\left|j_{\mu}(0)\right| 0\right\rangle \\
& =F_{\pi A_{1} \gamma}^{1}\left[\epsilon_{\mu}^{\lambda_{b}^{*}} q^{2}-\epsilon^{\lambda_{b}^{*}} \cdot q q_{\mu}\right]+F_{\pi A_{1} \gamma}^{2}\left[\epsilon_{\mu}^{\lambda_{b}^{*}} p_{a} \cdot q-\epsilon^{\lambda_{b}^{*}} \cdot q p_{a \mu}\right](4.38)
\end{aligned}
$$

Clearly (4.38) obeys current conservation. Furthermore the ansatz (4.38) is not unique, as it was the case for $\pi \omega$ and $\pi \mathrm{A}_{2}$. The helicity form factors for this ansatz are:

$$
\begin{gathered}
\Gamma^{0,0}=\frac{\sqrt{q^{2}}}{2 m_{A_{1}}}\left(F_{\pi A_{1} \gamma}^{1}\left(q^{2}+m_{A_{1}}^{2}-m_{\pi}^{2}\right)+F_{\pi A_{1} \gamma}^{2}\left(q^{2}-m_{A_{1}}^{2}-m_{\pi}^{2}\right)\right) \\
\Gamma^{0, \lambda_{b}}=F_{\pi A_{1} \gamma}^{1} q^{2}+F_{\pi A_{1} \gamma}^{2} \frac{1}{2}\left(q^{2}-m_{A_{1}}^{2}+m_{\pi}^{2}\right), \\
\lambda_{b}= \pm 1
\end{gathered}
$$


We notice that the longitudinal matrix element $\Gamma^{0,0}$ vanishes for $\mathrm{q}^{2}=0$, as it should be. The coefficient of $\epsilon_{\mu}^{\lambda_{b}^{*}}$, namely $F_{\pi A_{1} \gamma}^{1} q^{2}+F_{\pi A_{1} \gamma}^{2} p_{a} \cdot q$ is the transverse form factor. For the decay $A_{1} \rightarrow \pi \gamma$ only $F_{\pi A_{1} \gamma}^{2}$ contributes. The cross section for $\pi A_{1}$ production is according to (4.17):

$$
\frac{d \sigma}{d \cos \theta}=\frac{\pi \alpha^{2}}{2 W^{4}}\left(\frac{p}{W}\right)\left\{\sin ^{2} \theta\left|\Gamma^{0,0}\right|^{2}+\left(1+\cos ^{2} \theta\right) /\left.\Gamma^{0,1}\right|^{2}\right\}
$$

with $\Gamma^{0,0}$ and $\Gamma^{0,1}$ given by (4.39). By measuring the coefficients of $\sin ^{2} \theta$ and $\left(1+\cos ^{2} \theta\right)$ one can determine the absolute values of two linear combinations of the invariant form factors $F_{\pi A_{1} \gamma}^{1}$ and $F_{\pi A_{1} \gamma}^{2}$. To determine their relative phase one has to measure the polarization of the $A_{1}$. This will be discussed later.

It is obvious that the equations $(4.38),(4.39)$ and $(4.40)$ with appropriate change of notation are also valid for the final states $\pi B, \eta B, \varepsilon \rho, \varepsilon \omega, \varepsilon \varphi, \delta \rho, \delta \omega, \delta \varphi$.

The most interesting case for fermion-antifermion production are $p \bar{p}$ and $n \bar{n}$ final states. The current matrix element in covariant form is well known and is given by two invariant form factors $f_{1}$ and $f_{2}$ : 


$$
\begin{aligned}
& (2 \pi)^{3}\left\langle N\left(p_{a}\right), \bar{N}\left(p_{b}\right)\left|j_{\mu}(0)\right| 0\right\rangle= \\
& \bar{u}\left(p_{a}, \lambda_{a}\right)\left\{f_{1} \gamma_{\mu}+f_{2} / 2 m i \sigma_{\mu \nu} q^{\nu}\right\} V\left(p_{b}, \lambda_{b}\right)
\end{aligned}
$$

The helicity form factors corresponding to (4.41) are $\left(\eta=9^{2} / 4 \mathrm{~m}^{2}\right)$

$$
\begin{aligned}
& \Gamma^{\frac{1}{2}, \frac{1}{2}}=-2 m\left(f_{1}+\eta f_{2}\right) \equiv-2 m f_{c} \\
& \Gamma^{\frac{1}{2},-\frac{1}{2}}=\sqrt{2 q^{2}}\left(f_{1}+f_{2}\right) \equiv \sqrt{2 q^{2}} f_{m}
\end{aligned}
$$

The longitudinal form factor is the traditional electric form factor $f_{c}$ and the transverse form factor is the magnetic form factor $f_{m}$. The cross section is obtained with ( 3.17 to be:

$$
\frac{d \sigma}{d \cos \theta}=\frac{\pi \alpha^{2}}{W^{2}}\left(\frac{p}{w}\right) \frac{4 m^{2}}{W^{2}}\left\{\sin ^{2} \theta\left|f_{c}\right|^{2}+\left(1+\cos ^{2} \theta\right) \eta\left|f_{m}\right|^{2}\right\}
$$

\section{E. Vector Dominance Model for Form Factors and Comparison} with Experiment

In this paragraph we consider simple models for the invariant form factors in the timelike region $q^{2}>0$ for some special interesting cases as for example the pion form factor, the $\pi \omega$, 
$\pi A_{2}$ and $\pi A_{1}$ transition form factors. Let us start with the pion form factor $F_{\pi}$. We know $F_{\pi}\left(q^{2}\right)$ is the boundary value of an analytic function $F(z)$.

$$
F_{\pi}\left(q^{2}\right)= \begin{cases}\lim _{\varepsilon \rightarrow+\sigma} F\left(q^{2}+i \varepsilon\right) & q^{2} \geqslant 4 m_{\pi}^{2} \\ F\left(q^{2}\right) & q<4 m_{\pi}^{2}\end{cases}
$$

$F(z)$ is an analytic function of $z$ in the cut $z$ plane, the cut starting from $4 \mathrm{~m}_{\pi}^{2}$ to $+\infty$ on the real positive axis. If in the unitarity relation for $\operatorname{Im} F_{\pi}\left(q^{2}\right)$ only the $\pi \pi$ intermediate state is taken into account, (see Fig. 8) the phase of $F_{\pi}\left(q^{2}\right)$ is given by the phase shift $\delta_{1}$ of the elastic $\pi \pi$ scattering amplitude in the $p$-wave state. If furthermore $F(z)$ has no zeros in the complex $z$-plane, $F_{\pi}\left(q^{2}\right)$ can be expressed solely by the $J=1$ phase shift. The representation with the analytic properties stated above is then:

$$
F_{\pi}\left(q^{2}\right)=\exp \left\{\frac{q^{2}}{\pi} \int_{4 m_{\pi}^{2}}^{\infty} d x \frac{\delta_{1}(x)}{x\left(x-q^{2}-i \varepsilon\right)}\right\}
$$

Clearly $F_{\pi}(0)=1$. Unfortunately $\delta_{1}(x)$ is not well known, so we must compute it $V^{\text {in }}$ model dependent way. A model, which incorporates the $\rho$ resonance, i.e. $\delta_{1}\left(m_{\rho}^{2}\right)=\pi / 2 \quad$ but with zero width, is the following approximation for the phase shift: 
- $448-$

$$
\delta_{1}(x)=\left\{\begin{array}{lll}
0 & \text { for } & 4 m_{\pi}^{2} \leqslant x<m_{\rho}^{2} \\
\pi & \text { for } & m_{\rho}^{2}<x<\infty
\end{array}\right.
$$

This model, if substituted into (4.44) leads to the zero width $\rho$ dominance approximation

$$
F_{\pi}\left(q^{2}\right)=\frac{m_{\rho}^{2}}{m_{\rho}^{2}-q^{2}-i \varepsilon}
$$

Although this model is useful for spacelike $q^{2}$ it is inadequate for timelike $q^{2}$ because of the finite width of the $\rho$. There exist several models for $\delta_{1}\left(q^{2}\right)$ in the literature, which take into account the finite width of the $\rho$ and have correct threshold properties concerning the $p$-wave phase shift $\delta_{1}$.

Some of these models can be summarized by starting with a generalized effective range formula for $\delta_{1}\left(w^{2}\right)$

$$
\frac{k^{3}\left(W^{2}\right)}{W} \operatorname{ctg} \delta_{1}\left(W^{2}\right)=k^{2} h\left(W^{2}\right)+a+b W^{2}
$$

where

$$
h(x)=\frac{2}{\pi} \frac{k(x)}{\sqrt{x}} \ln \frac{2 k(x)+\sqrt{x}}{2 m_{\pi}}
$$

and $k(x)=\frac{1}{2}\left(x-4 m_{\pi}^{2}\right)^{1 / 2} \quad$ is the com. momentum of the $\pi$. The constants $a$ and $b$ are related to the $\rho$ meson parameters $m_{\rho}^{2}$ 
and $\Gamma_{\rho}$. Even if $\delta_{1}(x)$ is specified by (4.46), there is still the ambiguity of the asymptotic behaviour of $F_{\pi}$. In the simplest version, developed by Gounaris and Sakurai, $F_{\pi}^{-1}$ and $\frac{k^{3}}{W} \operatorname{ctg} \delta_{1}$ behave the same for $q^{2} \equiv W^{2} \rightarrow \infty$. (For other versions see (ref. 16) Frazer and Fulco, Vaughn and Wali, Roos and Pišut). Then $F_{\pi}$ is:

$$
F_{\pi}=\frac{m_{\rho}^{2}\left(1+d_{\rho} \Gamma_{\rho} / m_{\rho}\right)}{m_{\rho}^{2}-q^{2}+\frac{m_{\rho}^{2} T_{\rho}}{k_{\rho}^{3}}\left[k^{2}\left(h\left(q^{2}\right)-h_{\rho}\right)+\left(m_{\rho}^{2}-q^{2}\right) k_{\rho}^{2} h_{\rho}^{\prime}\right]-i m_{\rho} T_{\rho} \frac{k^{3}}{k_{\rho}^{3}} \frac{m_{\rho}}{\sqrt{q^{2}}}}(4.47)
$$

with $k_{\rho}=k\left(m_{\rho}^{2}\right)$ and $h_{\rho}=h\left(m_{\rho}^{2}\right)$. The numerical value of $d_{\rho}$ is 0.48 . (The Frazer-Fulco version is practically identical to $(4.47)$ as long as $\left|\mathrm{q}^{2}\right| \ll 9.610^{6} \mathrm{mg}^{2}$, the latter value is the zero of $F_{\pi}$ in the spacelike region). A fit to recent data for $e^{+} e^{-} \rightarrow \pi^{+} \pi^{-}$from the ACO storage ring in the $\rho$ resonance region yields the following parameters ${ }^{17}$ )

$$
\begin{aligned}
& m_{\rho}=(780.2 \pm 5.9) \mathrm{MeV}, \quad \Gamma_{\rho}=(152.8 \pm 15.1) \mathrm{MeV} \\
& g_{\rho}^{2} / 4 \pi=g_{\rho \pi \pi^{2}} / 4 \pi=2.56 \pm 0.22 \\
& \sigma_{\text {peak }}\left(e^{+} e^{-} \rightarrow \pi^{+} \pi^{-}\right)=(0.96 \pm 0.09) \mu b, d_{\rho}=0.66 \pm 0.37
\end{aligned}
$$

The fit of the data in terms of $\left|F_{\pi}\right|^{2}$ obtained from the cross section by (4.29) is shown in Fig. 9. Actually a more complicated formula than (4.47) must be used to make a fit, since through 
higher order electromagnetic effects the $w$ resonance is mixed in. If this $w \rho$ interference is taken into account one obtains the parameters given above (the branching ratio $B_{\omega \rightarrow 2 \pi}$ is $(0.2 \pm 0.05)^{2}$ and the interference angle is $(87.5 \pm 15.4)^{\circ}$, close to $90^{\circ}$ as to be expected). 17)

At Novosibirsk and with the Adone ring at Frascati $\pi^{+} \pi^{-}$cross sections have been measured recently between 1.0 and $2.0 \mathrm{GeV}$ total energy. It is interesting to compare these results with the Gounaris-Sakurai formula. In Fig.10 we plotted the theoretical curve for $\left|F_{\pi}\right|^{2}$ with $d_{\rho}=0.66$ and compared it with these data. It seems that systematically the experimental values are larger than the predicted values. But we have to keep in mind, that, except for the VEEP (Novosibirsk) results, no separation of $\mathrm{K}^{+} \mathrm{K}^{-}$and $\pi^{+} \pi^{-}$ events has been carried out in the experiments. So, the above results in the case of the Frascati experiments refer to the sum of the $K K$ and $\pi \pi$ channel rather than the $\pi \pi$ channel alone. Furthermore the results of the two Frascati groups do not agree terribly well with each other. On theoretical ground we expect deviations from the Gounaris-Sakurai expression for large W through admixtures of inelastic states in the unitarity relation like $k \bar{K}, \pi \omega, 4 \pi, \pi A_{1}$, $\bar{p} p$ and $\bar{n} n$ (see for example Renard ${ }^{18}$ ), Brunila, Roos and Pisút 19) ). Unfortunately an evaluation of such inelastic contributions to $F_{\pi}$ for larger $W^{2}$ has not been done yet. Furthermore we should notice that $t^{\text {the }}$ absolute event rates for the points reported are rather small. The VEEP-points (Novosibirsk) come 
from 12 events, the $\mu \pi$ group has $39 \pi^{+} \pi^{-}$events and the Adone BCF-group measured 41 events.

Finally we mention that if the Orsay data are fitted without finite width corrections, that means with $d_{\rho}=0$, the pion form factor has the form

$$
F_{\pi}=\frac{m_{\rho}^{2} g_{\rho \pi \pi}}{g_{\rho}\left(m_{\rho}^{2}-q^{2}-i m_{\rho} \Gamma\left(q^{2}\right)\right)}
$$

where $\Gamma\left(q^{2}\right)$ is the mass dependent width

$$
\Gamma\left(q^{2}\right)=\Gamma_{\rho} \frac{k^{3}\left(q^{2}\right) m_{\rho}}{k_{\rho}^{3} \sqrt{q^{2}}}
$$

with $\Gamma_{\rho}=\frac{2}{3} g_{\rho \pi \pi}{ }^{2} / 4 \pi \cdot \mathrm{kg}_{\rho}^{3} / \mathrm{m}_{\rho}^{2}$ and $1 / g_{\rho}$ is the photon-rho coupling. Ore obtains the best fit for $\Gamma_{\rho}=(149.6 \pm 23.2) \mathrm{MeV}$, $\phi=(85.7 \pm 15.3)^{\circ}, m_{\rho}=(775.4 \pm 7.3) \mathrm{MeV}, g_{9 \pi}^{2} / 4 \pi=(2.84 \pm 0.50)$ and $g_{s}^{2} / 4 \pi=(2.26 \pm 0.25)$, compared to $g_{\rho}^{2} / 4 \pi=g_{\rho \pi \pi}^{2} / 4 \pi=(2.56$ $\pm 0.22)$ with finite width corrections. Next we consider the $\pi^{\circ} \omega$ transition form factor. Unfortunately a dynamical calculation similar to the pion form factor has not been done yet. In this case one has to solve the coupled channel problem $\pi \pi \rightarrow \pi \pi$ and $\pi \pi \rightarrow \pi \omega$ and calculate from it the 
electromagnetic transition form factor. Instead we shall start from the $\rho$ dominance assumption and apply it to the invariant form factor $F_{\pi \omega \gamma}$ defined in (4.30). The result is:

$$
F_{\pi \omega \gamma}\left(q^{2}\right)=\frac{F_{\pi \omega \rho} m_{\rho}^{2}}{g_{\rho}\left(m_{\rho}^{2}-q^{2}-i m_{\rho} \Gamma\left(q^{2}\right)\right)}
$$

where $F_{\pi \omega}$ is the invariant coupling between $\pi \omega \rho$ defined analogously to $F_{\pi \omega \gamma}$ in (4.30). It is to be expected, that, because of the larger spin of the w compared to the pion, the transition form factor falls off somewhat stronger with $q^{2}$ than given by $(4.50)$. But since experimentally $F_{\pi}$ seems to fall off less than given by $\rho$ dominance, (4.50) might be a reasonable approximation. The value for $g_{\rho}$ was given above. $F_{\pi \omega \rho}$ can be calculated from the $\omega \rightarrow 3 \pi$ decay. If we use the model of Goldberg and Srivastava, which is a modified $\rho$ pole model, we have from $\Gamma_{\omega \rightarrow 3 \pi}=8.97 \mathrm{MeV}$, which is the most recent value for the $3 \pi$ decay width of the $\omega$, the value $F_{\pi \omega \rho}^{2} / 4 \pi=26.4 \mathrm{GeV}^{-2}$ (This leads to $\Gamma_{\omega \rightarrow \pi \gamma}=1.50 \mathrm{MeV}$, compared to the experimental value $\left.\Gamma_{\omega \rightarrow \pi \gamma}=(0.9 \pm 0.1) \mathrm{MeV}\right)$. With these coupling constants we get for the reaction $\mathrm{e}^{+} \mathrm{e}^{-} \rightarrow \pi^{0} \omega$ the cross section as shown in Fig. 11. Just above threshold ( $W=0.9 \mathrm{GeV})$ the cross section rises to its maximum around $10 \mathrm{nb}\left(1 \mathrm{nb}=10^{-33} \mathrm{~cm}^{2}\right)$ and then falls off slowly with $q^{2}$. 
We can apply the same formulas for the transition form factor $\pi^{0} \rho^{0}$ by replacing $g_{\rho}$ by $g_{\omega}$ and the masses appropriately. With $g_{\omega}^{2} / 4 \pi=18.4 \pm 2.0$ the curve labelled $\pi^{0} \rho^{0}$ is obtained for the cross section (see Fig. 11). This curve can be directly compared to experimental data, since at the energies, we consider, other two-particle states do not contribute to the final state $\pi^{+} \pi^{-} \pi^{0}$, which is the decay product of $\pi^{0} \rho^{0}, \pi^{+} \rho^{-}$and $\pi^{-} \rho^{+}$. The ACO results for the cross section $\pi^{+} \pi^{-} \pi^{0}$ at $0.99 \mathrm{GeV}$ are quoted as being in agreement with $3 \sigma\left(\pi^{\circ} \rho^{0}\right)$. The Adone $\gamma \gamma$ group obtained for $1.85 \leqslant W \leqslant 2.10 \mathrm{GeV}$ a value for $\sigma=(2 \pm 2) \mathrm{nb}$ from one specification of events and $\sigma \leqq(5+2) n b$ as upper limit for another class of events. These values are not inconsistent with the prediction $0.9 \mathrm{nb}$ for $\mathrm{W}=2.0 \mathrm{GeV}$. Concerning the $\pi^{\circ} \omega$ cross section we discuss the comparison with the experimental data after we calculated the other channels which contribute to the $\pi^{+} \pi^{-} \pi^{0} \pi^{0}$ final state. The calculation of the cross section for $\pi A_{2}$ is quite similar. The invariant form factor $F_{\pi A_{2}} \gamma$ is given by rho dominance

$$
F_{\pi A_{2} \gamma}\left(q^{2}\right)=\frac{F_{\pi A_{2} \rho} m_{\rho}^{2}}{g_{\rho}\left(m_{\rho}^{2}-q^{2}-i m_{\rho} \Gamma\left(q^{2}\right)\right)}
$$

The coupling $F_{\pi A_{2} \rho}$ is obtained from the $A_{2}$ width into $\rho \pi$ which is $(0.0768 \pm 0.0160 \mathrm{GeV}$. The relation is: 


$$
\Gamma_{A_{2} \rightarrow \rho \pi}=\frac{p_{\rho}}{20 \pi m_{A_{2}}^{2}}\left|\Gamma^{01}\right|^{2}=\frac{F_{\pi A_{2} \rho}^{2}}{4 \pi} \frac{p_{\rho}^{5}}{10}
$$

where $p_{s}$ is the momentum of the rho in the $A_{2}$ rest frame and $\Gamma^{01}$ is obtained from (4.36). The final formula for the cross section in terms of the partial decay width is:

$$
\sigma=86.5 g\left(\frac{p}{p_{\rho}}\right)^{5} \frac{5 \Gamma_{A_{2} \rightarrow \rho \pi}}{m_{A_{2}}^{2} W} \quad \frac{4 \pi}{g_{\rho}^{2}}\left|\frac{m_{\rho}^{2}}{m_{\rho}^{2}-q^{2}-i m_{\rho} \Gamma\left(q^{2}\right)}\right|^{2}
$$

$$
\sigma \text { in } n b \text {, other quantities in GeV. }
$$

For $\mathrm{q}^{2}$ large $\sigma$ goes to a constant in disagreement with the unitarity constraint $\sigma \ll \frac{1}{q^{2}}$. Therefore we must expect that additional $\eta^{2}$ dependence is present through coupling to other states. Without these additional form factors the cross section rises from threshold $(\mathrm{W}=1.5 \mathrm{GeV})$ to about $3 \mathrm{nb}$ at $\mathrm{W}=3 \mathrm{GeV}$ (see Fig.14).

For $\pi \mathrm{A}_{1}$ the calculation of the cross section in the $\rho$ dominance approximation proceeds as follows. For the two invariant form factors $F_{\pi A_{1} \gamma}^{1}$ and $F_{\pi A_{2} \gamma}^{2}$ we have:

$$
F_{\pi A_{1} \gamma}^{i}\left(q^{2}\right)=\frac{F_{\pi A_{1} \rho}^{i} m_{\rho}^{2}}{g_{\rho}\left(m_{\rho}^{2}-q^{2}-i m_{\rho} \Gamma\left(q^{2}\right)\right)} ; i=1,2
$$


The width of the $A_{1}$ yields one relation for these two coupling constants

$$
\Gamma_{A_{1}}=\frac{p_{s}}{24 \pi m_{A_{1}}^{2}}\left\{\left|\Gamma^{0,0}\right|^{2}+2\left|\Gamma^{0,1}\right|^{2}\right\}
$$

where $\Gamma^{0,0}$ and $\Gamma^{0,1}$ are identical to $(4.39)$ with $q^{2}=m_{\rho}^{2}$ and with the appropriate replacement of coupling constants

$$
F_{\pi A_{1} \gamma}^{i} \rightarrow F_{\pi A_{1} \rho}^{i} \quad \text {. We calculate the cross section, inte- }
$$

grated over angle, from formula (4.40) in terms of two constants, the width $\Gamma_{A_{1} \rightarrow \rho \pi}=0.1 \mathrm{GeV}$ and the ratio $y=\frac{F_{\pi A_{1} \rho}^{2}}{F^{1} \pi A_{1} \rho}$ of the two coupling constants at the $\rho$ mass. The result is seen in Fig. 12, where we present the cross section for $\pi \mathrm{A}_{1}$ production for $y=-1.0,0.0,1.0$ and 2.0. We see that the cross section depends sensitively on the ratio $y$. The cross section may be as large as several hundred nanobarn at its maximum around $W=1.4 \mathrm{GeV}$. To see this variation of the cross section at the peak we plotted $\sigma$ for $W=1.409 \mathrm{GeV}$ and $W=2.209 \mathrm{GeV}$ as a function of $y$ in Fig.13. The possible maximum under the constraint $\Gamma_{A_{1}}=0.1 \mathrm{GeV}$ is for $y=2.2$. The reason for this strong variation of $\sigma$ is the dependence of the width $\Gamma_{A_{1}}$ on $y$. $\Gamma_{A_{1}}$ has as a function of $y$ a minimum around this value, which leads to a large coupling constant $\left(F_{\pi A_{1} \rho}^{1}\right)^{2} / 4 \pi$ in this case. $y$ can be measured from the decay density matrix. From a recent measure24) ment we have for the ratio of decay matrix elements $g_{\lambda_{\rho}}\left(\lambda_{\rho}=0,1\right)$ 
in the rest system of the $A_{1}$

$$
0.68 \leqslant g_{1} / g_{0} \leqslant 1.6
$$

$g_{0}$ and $g_{1}$ are related to the s-wave to d-wave decay coupling in the following way:

$$
g_{d} / g_{s}=\sqrt{2} \frac{g_{1} / g_{0}-1}{2 g_{1} / g_{0}+1}
$$

Thus the value given in $(4.56)$ is consistent with no d-wave contribution in the decay. The relation of $g_{1} / g_{0}$ with $y$ is as follows:

$$
g_{1} / g_{0}=\frac{m_{\rho}\left(2 m_{\rho}^{2}-y\left(m_{A_{1}}^{2}-m_{\rho}^{2}-m_{\pi}^{2}\right)\right)}{2 m_{A_{1}} p_{\rho}^{2} y}
$$

which leads to the following limits for $y, 1.5 \leqslant y \leqslant 1.9$. No d-wave corresponds to $y=1.7$. Thus with $y=1.7$ the $\pi \mathrm{A}_{1}$ cross section should be rather large, around $100 \mathrm{nb}$ at the peak. Unfortunately this comparison of $y$ with the decay matrix elements cannot be taken too seriously. First there are problems in the experimental determination of $y$ itself (background subtractions, assumptions about production mechanism). vecond with our ansatz 
in (4.38) we propose a special ansatz for extrapolation from $W^{2}=m_{\rho}^{2}$ to $W^{2}$ above threshold $(W=1.4 \mathrm{GeV})$. Other assumptions about the definitions of invariant form factors may lead to different relations between coupling constants and $g_{1} / g_{0}$ than those in ( 4.58). Finally we mention that the value of $y$ has also influence on the $\mathrm{W}^{2}$ dependence of the cross section. Decreasing $y$ below $y=2.2$ leads to smaller cross sections and stronger fall off with $W^{2}$ (see Fig.13).

Another important channel is $\varepsilon \rho$, where $\varepsilon$ is the broad $0^{+}$ resonance around $.750 \mathrm{GeV}$. The formulas are the same as for the $\pi A_{1}$ channel with the replacement $\pi \rightarrow \varepsilon, A_{1} \rightarrow \rho$. Since $\rho$ and $\varepsilon$ have almost equal masses $F_{\varepsilon \rho \rho}^{i}(i=1,2)$ can be obtained only indirectly. $F_{\varepsilon \rho \rho}^{2}$ is related by rho dominance to the $\varepsilon \rightarrow 2 \gamma$ decay. This has not been measured. But there exist calculations for the partial width of the $\varepsilon$ into two photons on the basis of a dispersion theoretical model. We use this result $\quad \Gamma_{\varepsilon \rightarrow 2 \gamma}=27.4 \mathrm{KeV}$ as a constraint for our calculation. The relation between this width and $F_{E \rho \rho}^{2}$ is:

$$
\Gamma_{\varepsilon \rightarrow 2 \gamma}=\left(\frac{\alpha}{g_{\rho}^{2} / 4 \pi}\right)^{2} \cdot \frac{\left(F_{\varepsilon \rho \rho}^{2}\right)^{2}}{4 \pi} \cdot \frac{m_{\epsilon}^{3}}{16}
$$

As second parameter we choose $x=F_{\varepsilon \rho \rho}^{1} / F_{\varepsilon \rho \rho}^{2}$. The result for $\sigma_{\varepsilon \rho}$ is given in Fig.14. The cross section is appreciable, 
around $30 \mathrm{nb}$ for $x=0$ at the maximum. The threshold is at $W=1.52 \mathrm{GeV}$. The cross section depends sensitively on $x$. It rises with $x$ positive and has a minimum around $x=-0.6$, where also the $W^{2}$ dependence is changed (the maximum occurs around $W=3.0 \mathrm{GeV}$ instead of $W=1.7 \mathrm{GeV}$ for the other $x$ values), and increases again if $x$ becomes more negative. For $x=-1.0$ the cross section falls off with $w^{2}$ stronger than for $x=1.0$, although at the maximum the values for $\sigma$ are about the same.

It is obvious that we get similar results for the final states $\pi^{\circ} \mathrm{B}^{\circ}$ and $\pi^{ \pm} \mathrm{B}^{\mp}$ as for $\pi \mathrm{A}_{1}$ except that the intermediate state is now the $w$ instead of the $\rho$. If the masses of $B$ and $A_{1}$ would be equal the cross section for $\pi^{\circ} B^{\circ}$ with the same value for $y$, would be just one tenth of the $\pi^{+} \mathrm{A}_{1}+\pi^{-} \mathrm{A}_{1}$ cross section since $g_{\omega}^{2} \approx 10 g_{\rho}^{2}$. Since the mass of the B is larger $\left(m_{B}^{2}=1.52 \mathrm{GeV}^{2}\right.$, $m_{A_{1}}^{2}=1.14 \mathrm{GeV}^{2}$ ) the maximum of the peak cross section occurs for smaller values of $y$, around $y=1.5$ and is only about $10 \mathrm{nb}$. In Fig. 15 we present the cross section for $\mathrm{W}=1.612 \mathrm{GeV}$, where the cross section has its maximum, and for $W=2.412$ as a function of $y$ (the threshold is at $W=1.372 \mathrm{GeV}$ ). $y$ is defined in the same way as in the case of $\pi \mathrm{A}_{1}$. From this we conclude that the total $\pi B$ contribution (all three channels) can be as high as $30 \mathrm{nb}$ around $W \approx 1.6 \mathrm{GeV}$. The decay of $B$ into $\pi \omega$ is a mixture of $s$ and $d$ waves. The limit for $g_{1} / g_{0}$ reported in the literature is 26):

$$
0.0 \leqslant g_{0} / g_{1} \leqslant 0.68
$$


These limits lead to: $0 \leqslant y \leqslant 0.82$. This indicates that the total $\pi B$ contribution may be less than the $30 \mathrm{nb}$ at the maximum .

The $\omega$ is also coupled to $\varepsilon \omega$ and $\varepsilon \varphi$. The contribution of these final states is similar to $\varepsilon \rho$. Its magnitude depends on the $\varepsilon \omega \omega, \varepsilon \varphi \omega$ and $\varepsilon \varphi \varphi$ coupling. No calculations are available about these couplings. Also symmetry estimates are rather uncertain, since the mixing in the $0^{+}$nonet is not known. If we assume that the $\varepsilon$ is the singlet member of the nonet we have

$$
\left\langle\varepsilon \omega\left|g_{\mu}(0)\right| 0\right\rangle \approx \frac{1}{3}\left\langle\varepsilon \rho\left|g_{\mu}(0)\right| 0\right\rangle \text { and }\left\langle\varepsilon \varphi\left|\jmath_{\mu}(0)\right| 0\right\rangle \approx-\frac{\sqrt{2}}{3}\left\langle\varepsilon \rho\left|g_{\mu}(0)\right| 0\right\rangle
$$

Thus the contributions of these final states seem to be smaller than $E \rho$. Better estimates are only possible after studying the mixing in the $0^{+}$nonet and after taking into account also breaking effects.

As our last example we consider the production of $\rho^{+} \rho^{-}$. It is described by three form factors $f c, f m, f q$, the charge, magnetic and quadrupole form factor. The cross section depends on the relation of these three form factors. In Fig. 10 we show the integrated cross section for three cases: 1) $f m=f c, f q=0$; 2) $f_{m}=2 f_{c}, f_{q}=0$; 3) $f_{c}=f_{q}=0.5 f_{m}$, where $f_{c}$ is given by the $\rho$ dominance expression $f_{c}=\frac{m_{g}^{2}}{m_{g}^{2}-q^{2}-i m_{g} \Gamma}$ $f_{c}(0)=1$, because of normalization of charge. Some formulations of current algebra predict $f_{m}=2 f_{c}, f_{q}=0$. We see that the 
cross section may vary by an order of magnitude, depending on the relation of $f_{m}$ and $f_{q}$ to $f_{c} \cdot$ (See Fig. 16)

Now we come to the comparison of the cross section for resonance production with recent experimental data obtained at ADONE (Frascati) 27). Unfortunately the rate of many pion events is not so numerous, that resonances could be isolated in the final state. Up to now only total cross section for different charge states, sometimes with an unknown number of neutral pions have been reported. In the process $\mathrm{e}^{+} \mathrm{e}^{-} \rightarrow \pi^{+} \pi^{-} \pi^{+} \pi^{-}$evidence against a pure phase space production seems to be established. It looks that this final state is very much $\rho \pi \pi$, instead of four charged pions. Whether this final state has a large $\pi A_{1}$ signal is not clear at present, although the plot of the invariant mass of three pions seems to show a maximum in the vicinity of $1.1 \mathrm{GeV}$. (See Fig. 17, which has been taken from the report of Grilli ${ }^{2}$ )). The experimental results for $\sigma_{2, n}\left(e^{+} e^{-} \rightarrow \pi^{+} \pi^{-}+\right.$neutrals $)$, $\sigma_{4,0}\left(e^{+} e^{-} \rightarrow \pi^{+} \pi^{-} \pi^{+} \pi^{-}\right), \quad \sigma_{4, n}\left(e^{+} e^{-} \rightarrow \pi^{+} \pi^{-} \pi^{+} \pi^{-}+\right.$neutrals $)$and $\sigma_{\text {tot }}\left(e^{+} e^{-} \rightarrow \pi^{+} \pi^{-}+\right.$anything $)$are summarized in Fig. 18-21. If we attempt to explain these cross sections by pure resonance production we must sum the following terms for the different charge states:

$$
\begin{aligned}
& \pi^{+} \pi^{-} \pi^{0} \pi^{0}: \quad \pi \omega, \pi \varphi, \frac{1}{2}\left(\pi A_{1}\right), \frac{1}{2}\left(\pi A_{2}\right), \rho^{+} \rho^{-}, \frac{1}{3}(\varepsilon \rho), \frac{1}{3}(\rho f) \\
& \pi^{+} \pi^{-} \pi^{+} \pi^{-}: \quad \frac{1}{2}\left(\pi A_{1}\right), \frac{1}{2}\left(\pi A_{2}\right), \frac{2}{3}(\varepsilon \rho), \frac{2}{3}(\rho f)
\end{aligned}
$$




$$
\begin{aligned}
& \pi^{+} \pi^{-} \pi^{+} \pi^{-} \pi^{0}: \quad 2(\pi B), \frac{2}{3}(\varepsilon \omega), 2\left(\rho A_{2}\right), 2\left(\rho A_{1}\right), \frac{2}{3}(\omega f) \\
& \pi^{+} \pi^{-} \pi^{0} \pi^{0} \pi^{0}: \quad \pi B, \quad \frac{1}{3}(\varepsilon \omega), \rho A_{2}, \rho A_{1}, \frac{1}{3}(\omega f) \\
& \pi^{+} \pi^{-} \pi^{+} \pi^{-} \pi^{0} \pi^{0}: \quad \omega A_{2}, \omega A_{1}, 2(\rho B), \frac{2}{3}(\varepsilon B), \frac{1}{2}\left(A_{1} A_{1}\right) \\
& \pi^{+} \pi^{-} \pi^{0} \pi^{0} \pi^{0} \pi^{0}: \quad \frac{1}{3}(\varepsilon B), \frac{1}{4}\left(A_{1} A_{1}\right) \\
& \pi^{+} \pi^{-} \pi^{+} \pi^{-} \pi^{+} \pi^{-}: \quad \frac{1}{4}\left(A_{1} A_{2}\right)
\end{aligned}
$$

In this table we inciuded also such final states, as for example ( $\rho f)$, which we have not discussed in the last section, and where very little is known about the cross sections to be expected. Most of these channels have thresholds above $\mathrm{W}=2.5 \mathrm{GeV}$. Therefore the main channels for $\pi^{+} \pi^{-} \pi^{+} \pi^{-}$are $\frac{1}{2}\left(\pi \mathrm{A}_{1}\right)$ and $\frac{2}{3}(\varepsilon \rho)$ and for $\pi^{+} \pi^{-} \pi^{\circ} \pi^{\circ}: \frac{1}{2}\left(\pi A_{1}\right), \frac{1}{3}(\varepsilon \rho)$ and $(\pi \omega)$. If we choose the following ratios of coupling constants: $y=1.0$ for $\pi A_{1}$ and $x=-1.0$ for $\varepsilon \rho$, then we obtain for these two channels the results shown in Fig. 22. In $\pi^{+} \pi^{-} \pi^{+} \pi^{-}$the major portion of the cross section is $\varepsilon \rho$, in order to keep the cross section for $\pi^{+} \pi^{-} \pi^{\circ} \pi^{\circ}$ down. We see that our resonance model can qualitatively explain the observed magnitude, al though the data for $\pi^{+} \pi^{-} \pi^{+} \pi^{-}$seem to fall off stronger with increasing $W$ than the model predicts 28 ). Furthermore the measured values for $\pi^{+} \pi^{-} \pi^{0} \pi^{0}$ seem to be lower than the calculated ones. We have to admit, that the resonance 
model is rather crude and needs improvement in several respects. First the zero-width approximation is rather doubtful, Second there are contributions caused by the interference of several resonances. This is particularly important for $\varepsilon \rho$. Third there are contributions from other intermediate states like $\pi$-exchange with poles outside the physical region. Furthermore it is to be expected, that the form factors in $\mathrm{q}^{2} \equiv \mathrm{w}^{2}$ are only very crudely approximated by the $\rho, w$ or $\varphi$ tails and that the $q^{2}$ dependence is stronger than given by these contributions. If we rely primariIy on the measured points of the $\mu \pi$ group (see Fig. 22), then the variation of the cross section for $\pi^{+} \pi^{-} \pi^{+} \pi^{-}$production with $W$ suggests the production of a resonance $\rho^{\prime}$ with mass around $1.6 \mathrm{GeV}$. This possibility has been considered in more detail by Bramon and Greco 29). They correlate the process $e^{+} e^{-} \rightarrow \pi^{+} \pi^{-} \pi^{+} \pi^{-}$with the reaction $\gamma p \rightarrow \pi^{+} \pi^{-} \pi^{+} \pi^{-}$, in which a $4 \pi^{ \pm}$enhancement centered at $(1.6 \pm 0.1) \mathrm{GeV}$ with a width $(0.5 \pm 0.1) \mathrm{GeV}$ and a cross section of $(0.9 \pm 0.2) \mu b$ seems to have been found recently 30). Furthermore they estimate the coupling $g_{\rho}^{\prime}$ of the $\rho^{\prime}$ to the photon from vector-meson-dominance relations based on $\rho, \omega, \varphi$ and $\rho^{\prime}$. With this $\left(g_{\rho^{\prime}} / g_{\rho}\right)^{2}=5$ and the partial decay width of $\rho^{\prime} \rightarrow 4 \pi^{ \pm}$obtained from the $\not p \rightarrow \pi^{+} \pi^{-} \pi^{+} \pi^{-}$cross section, they calculate the cross section $\sigma_{4 \pi^{ \pm}}$from:

$$
\sigma_{4 \pi^{ \pm}}=\frac{16 \pi^{2} \alpha^{2} m_{\rho^{\prime}}^{4}}{g_{\rho^{\prime}}^{2} W^{3}} \frac{\Gamma_{\rho^{\prime} \rightarrow 4 \pi^{ \pm}}}{\left(W^{2}-m_{\rho^{\prime}}^{2}\right)^{2}+m_{\rho^{\prime}}^{2} \Gamma_{\rho^{\prime}}^{2}}
$$


and find the curve in Fig. 23, where the shaded area gives the limit of the theoretical prediction. The calculated cross section at the maximum is $\sigma_{4 \pi^{ \pm}}=(16 \pm 5) n b$ and roughly fits the measured points. The total width $T_{\rho^{\prime}}$ was set equal to $0.35 \mathrm{GeV}$. Clearly better experimental data are needed before final judgement is possible.

\section{Conclusions and Outlook}

The results of the Frascati experiments show, that above $1 \mathrm{GeV}$ total energy $e^{+} e^{-}$annihilation consists mainly of multipion production with a cross section of the order of $100 \mathrm{nb}=10^{-31} \mathrm{~cm}^{2}$, slowly decreasing with total energy. In the last section we made the attempt to explain this large cross section by the summation of all quasi-two-body modes $\left(\pi+R: \pi A_{1}, \pi A_{2}, \pi B, \pi \omega \ldots\right.$ or $R+R^{\prime}$ : $\varepsilon \rho, \rho \rho, \ldots)$ coupled to the intermediary vector mesons $\rho, \omega$ and $\varphi$. For reasons of simplicity we restrained ourselves to the discussion of the particular channels $\pi^{+} \pi^{-} \pi^{0}, \pi^{+} \pi^{-} \pi^{0} \pi^{0}$ and $\pi^{+} \pi^{-} \pi^{+} \pi^{-}$. But it is clear that, because of the successive openings of the various $\pi+R$ and $R+R^{\prime}$ channels, the total cross section should be relatively constant or decrease only slowly with total energy. Up to now it is not certain whether these resonances are really produced with such large cross sections as predicted. But in any case, because of the large experimental cross sections, the $\mathrm{e}^{+} \mathrm{e}^{-}$annihilation into many pions will be a very important tool for studying pion-pion dynamics. Actually the process 
$\mathrm{e}^{+} \mathrm{e}^{-} \rightarrow \pi_{1}, \pi_{2}, \ldots, \pi_{n}$ with $n$ pions in the final state is characteristically an amplitude for the decay of a vector particle (the virtual photon) with mass $q^{2}$ into $n$ pions. By varying the mass of the decaying photon one has the possibility to study the mutual $\pi \pi$ interactions in different energy regions, low and high energies. At low energies resonance phenomena should dominate whereas at higher energies characteristic asymptotic properties of the amplitudes should evolve. Using sum rules one has connections between the resonance region and the asymptotic region. This has been studied for example for the one-particle distribution functions by Renard 31). of course there are many more interesting properties of multipion final states which can be studied as a function of $q^{2}$, as for example, the multiplicity, the charge distribution and the mean momentum of the pions. 33) 
References :

1) Earlier reviews of the theoretical aspects can be found in: N. Cabibbo and R. Gatto, Phys. Rev. 124, (1961), 1094 R. Gatto, in Proceedings of the International Symposium on Electron and Photon Interactions at High Energies, Hamburg 1965 (Springer, Berlin, 1965), p. 106 and Springer Tracts in Modern Physics Vol. 39, (1965), p. 106.

M. Gourdin, Proceedings of the Eleventh Session of the Scottish Universities Summer School in Physics 1970, Hadronic Interactions of Electrons and Photons (Academic Press, London and New York, 1971), p. 395.

R. Gatto, Proceedings of the 4th International Symposium on Electron and Photon Interactions at High Energies, Liverpool 1968, p. 235.

D. Schildknecht, H.J. Willutzki and G. Wolf, DESY report $71 / 28$, June 1971.

2) Recent reviews of the experimental results are found in: J. LeFrancois, Proceedings of the 1971 International Symposium on Electron and Photon Interactions at High Energies (Cornell University, Ithaka, N.Y. (1971) ) p. 51. V.A. Sidorov, Proceedings of the 1971 International Symposium on Electron and Photon Interactions at High Energies (Cornell University, Ithaka, N.Y. (1971) ) p. 65.

Carlo Bernardini, Proceedings of the 1971 International Symposium on Electron and Photon Interactions at High Energies (Cornell University, Ithaka, N.Y. (1971) ) p. 37. M. Grilli (Lectures delivered at the "International School of Yerevan Physical Institute", November 23 - December 4, 1971), Frascati preprint INF 71/100, Dec. 1971. 
3) For example the reviews:

S. Brodsky, Proceedings of the 1971 International Symposium on Electron and Photon Interactions at High Energies (Cornell University, Ithaka, N.Y. (1971)) p. 13.

D. Schildknecht, H.J. Willutzki and G. Wolf, DESY report $71 / 28$, June 1971.

4) C. Bacci, G. Penso, G. Salvini, B. Stella, R. Baldini-Celio, G. Capon, C. Mencuccini, G.P. Murtas, A. Reale, M. Spinetti, Phys. Lett. 38B, (1972), 551.

G. Barbarino, F. Ceradini, M. Conversi, M. Grilli, E. Iarocci, M. Nigro, I. Paoluzi, R. Santonico, P. Spillantini, I. Trasatti, V. Valente, R. Visentin and G.T. Zorn, Frascati preprint INF 71/96, Dec. 1971. M. Grilli, see ref. 2 .

5) N. Cabibbo, G. Parisi, M. Testa, Lett. Nuovo Cim. 4, (1970), 35

6) For a similar model see: F.M. Renard, preprint, University of Montpellier PM/72/2

7) J.D. Bjorken, Phys. Rev. 148, 1467 (1967)

8) V.N. Gribov, B.I. Ioffe and I. Ya. Pomeranchuk, Phys. Lett. 24B, 554, (1967).

9) R. Gatto, Proceedings of the 4th International Symposium on Electron and Photon Interactions at High Energies, Liverpool 1968, p. 235 .

10) Taken from J. Le Francois, ref. 2.

11) S.D. Drell, D.J. Levy, T.-M. Yan, Phys. Rev. D1, 1617 (1970). 
12) Such models have been considered in the past by: Shui-Yin-Lo, Phys. Rev. 148, (1966), 1431.

G. Altarelli, S. de Gennaro, E. Celeghini, G. Longhi,

R. Gatto, Nuovo Cim. 47A, (1967), 113.

F.M. Renard, Nuovo Cim. 64, (1969), 979.

G. Kramer, J. Uretsky, T. Walsh, Phys. Rev. 3D, 719 (1971).

M. Vaughn, P. Polito, Lett. Nuovo Cim. 1, (1971), 74 .

J. Layssac, F. Renard, Lett. Nuovo Cim. 1 (1971), 197.

A.M. Altukhov and I.B. Khriplovich, Novosibirsk preprint, 1971.

F. Renard, Nuovo Cimento 6A, 134 (1972).

13) Similar analysis for electron scattering form factors in the Breit system has been done by

L. Durand III, P.C. De Celles and R.B. Marr, Phys. Rev. 126, 1882 (1962).

M. Gourdin, Nuovo Cim. 36, 129 (1965).

14) W.R. Theis and P.H. Hertel, Nuovo Cim. 66A, 152 (1970).

15) G. Gounaris and J.J. Sakurai, Phys. Rev. Lett. 21 (1968), 244.

16) W.R. Frazer and J. Fulco, Phys. Rev. 117 (1960) 1503, 1609. M.T. Vaughn and K.C. Wali, Phys. Rev. Lett. 21, (1968), 938, Phys. Rev. 177, (1969), 2199.

Matts Roos and Ján Pisút: Nucl. Phys. B10, (1969), 563.

17) D. Benaksas, G. Cosme, B. Jean-Marie, S. Jullian, F. Laplanche, J. Le François, A.D. Liberman, G. Parrour, J.P. Repellin, G. Sauvage, Phys. Lett. 39B, (1972), 289.

18) F. Renard, Nucl. Phys. B15, 267 (1970).

19) T. Brunila, M. Roos and J. Pisút, Nucl. Phys. B9, 461 (1969). 
20) An estimate is given in

V.N. Baier and V.S. Fadin, JETP Lett. 15, (1972), 151.

21) H. Goldberg and Y. Srivastava, Phys. Rev. Lett. 22, 749 (1969); 22, 1232 ( $\mathrm{E})$ (1969).

It is a modified version of the model of M. Gell-Mann,

D. Sharp, and W. Wagner, Phys. Rev. Lett. $\underline{8}, 261$ (1962)

using the Veneziano model.

22) See the article of Le Francois, ref. 2 .

23) C. Bacci, G. Penso, G. Salvini, B. Stella, R. Baldini-Celio, G. Capon, C. Mencuccini, G.P. Murtas, A. Reale, M. Spinetti, Phys. Lett. 38B, (1972), 551.

24) G. Ascoli et al., Phys. Rev. Lett. 26, 929 (1971).

R. Rinando et al., Nuovo Cim. 5A, 239 (1971).

25) G. Schierholz and K. Sundermeyer, Nucl. Phys. 40B, 125 (1972). See also: B. Schrempp-0tto, F. Schrempp and T.F. Walsh, Phys. Lett. 36B, 463 (1971).

26) G. Ascoli et al., Phys. Rev. Lett. 20, 1411 (1968). A. Werbrouck et aI., Nuovo Cim. Lett. IV, 1267 (1970).

27) See ref. 4, the review of Grilli, ref. 2 and

G. Barbarino, F. Ceradini, M. Conversi, M. Grilli, E. Iarocci,

M. Nigro, I. Paoluzi, R. Santonico, P. Spillantini, L. Trasatti,

V. Valente, R. Visentin and G.T. Zorn, Frascati preprint LNF 71/96, Dec. 1971.

28) See also the recent data of the boson group at Adone:

B. Bartoii, F. Felicetti, G. Marini, A. Nigro, H. Ogren,

V. Silvestrini and F. Vanoli, preprint Frascati, LNF-71/91

(Dec. 1971). 
29) A. Bramon and M. Greco, preprint Frascati LNF-71/97 Dec. 1971. See also: A. Bramon and M. Greco, Lett. Nuovo Cimento 1, 739 (1971).

30) M. Davier, I. Derado, D.C. Fries, F.F. Lin, R.F. Mozley, A.C. Odian, W.P. Swanson, F. Villa and D. Yount, Contribution to Cornell Conference (1971).

31) J. Layssac and F.M. Renard, preprint, Montpellier University, $\mathrm{PM} / 71 / 10$.

32) P.V. Landshoff, Phys. Lett. 32B, 57 (1970).

33) J.D. Bjorken and S.J. Brodsky, Phys. Rev. D1, 1460 (1970). 
Figure Captions:

Fig. 1: Diagram for the reaction $\mathrm{e}^{-\mathrm{e}^{+}} \rightarrow \mathrm{e}^{-\mathrm{e}^{+}}+$hadrons.

Fig. 2: Initial $\mathrm{e}^{+} \mathrm{e}^{-}$states.

Fig. 3: Diagram for $e^{+} e^{-} \rightarrow$ hadrons in the one-photon approximation.

Fig. 4a: Production of hadron jets $\mathrm{H}_{1}$ and $\mathrm{H}_{2}$ by $\mathrm{e}^{+} \mathrm{e}^{-}$annihilation.

Fig. 4b: $\mathrm{e}^{+} \mathrm{e}^{-}$annihilation into hadrons through point coupling to protons.

Fig. 5: Cross section for $e^{+} e^{-} \rightarrow \mu^{+} \mu^{-}$for energies around the $\varphi$ mass. $\left.{ }^{2}\right)$

Fig. 6: Kinematically allowed regions for $\mathrm{e}^{+} \mathrm{e}^{-} \rightarrow \pi+$ anything and for inelastic electron-pion scattering.

Fig. 7: $\mathrm{e}^{+} \mathrm{e}^{-}$annihilation into two particles with momenta $p_{a}, p_{b}$, spins $s_{a}, s_{b}$, and helicities $\lambda_{a}$ and $\lambda_{b}$

Fig. 8: Imaginary part of pion form factor in the two-pion approximation. 
Fig. 9: Pion form factor with $\rho \omega$ mixing with measured points from Orsay 17)

Fig. 10: Pion form factor above $1 \mathrm{GeV}$ total energy.

Fig. 11: Calculated cross sections for $\pi^{0} \omega, \pi^{0} \rho^{0}$ and $\pi \pm A_{2} \mp$ in the $\rho$ dominance approximation.

Fig. 12: Calculated cross section for $\pi A_{1}$ in the $\rho$ dominance approximation as a function of $W$ and for various $y$.

Fig. 13: Peak cross section for $\pi A_{1}$ as a function of $y$.

Fig 14: Calculated cross section for $\varepsilon \rho^{\circ}$ in the $\rho$ dominance approximation for various $x$.

Fig 15: Peak cross section for $\pi^{0} \mathrm{~B}^{0}$ in the $\omega$ dominance approximation as a function of $y$.

Fig. 16: Theoretical cross section for $\rho^{+} \rho^{-}$in the $\rho$ dominance approximation for various models.

Fig. 17a: Momentum distribution of produced pions from $e^{+} e^{-} \rightarrow \pi^{+} \pi^{-} \pi^{+} \pi^{-}$. The experimental distribution is compared to that expected in the case of a phase space production. The total energy is $2 \mathrm{E}=1.5 \mathrm{GeV}$. (See Grilli, ref. 2). 
Fig. 17b: Invariant mass of pions two by two $M(\pi \pi)$,

invariant mass three by three $M(\pi \pi \pi)$, in events $e^{+} e^{-} \rightarrow \pi^{+} \pi^{-} \pi^{+} \pi^{-}$at $2 E=1.5 \mathrm{GeV}$. The experimental

distribution is compared with the phase space

calculation for four pions. (See Grilli, ref. 2).

Fig. 18: Cross section for processes, in which only two charged pions plus neutral pions are produced $\left(\sigma_{2, n}\right)$ for various total energies $2 \mathrm{E}$.

Fig. 19: Cross sections for the processes $e^{+} e^{-} \rightarrow \pi^{+} \pi^{-} \pi^{+} \pi^{-}\left(\sigma_{4,0}\right)$ and $e^{+} e^{-} \rightarrow \pi^{+} \pi^{+} \pi^{+}+$ $(1-2) \pi^{0}\left(\sigma_{4, n}\right)$ vs. $2 \mathrm{E}$.

Fig. 20: Cross section for processes in which at least four charged pions have been produced $\sigma_{\geqslant 4}=\sigma_{4,0}+\sigma_{4, n}+\sigma_{6,0}$

Fig. 21: Total multi-hadronic cross section vs. 2E. It is the cross section for processes in which more than two pions have been produced.

Fig 22: Comparison of resonance model for $\pi^{+} \pi^{+} \pi^{-}$and $\pi^{+} \pi^{-} \pi^{0} \pi^{0}$ production, based on $\varepsilon \rho^{0}$ and $\pi A_{1}$, with experimental data from ACO and various Adone groups.

Fig. 23: Prediction of $\rho^{\prime}$ resonance model for $\pi^{+} \pi^{-} \pi^{+} \pi^{-}$ production 29) and comparison with experimental data. 


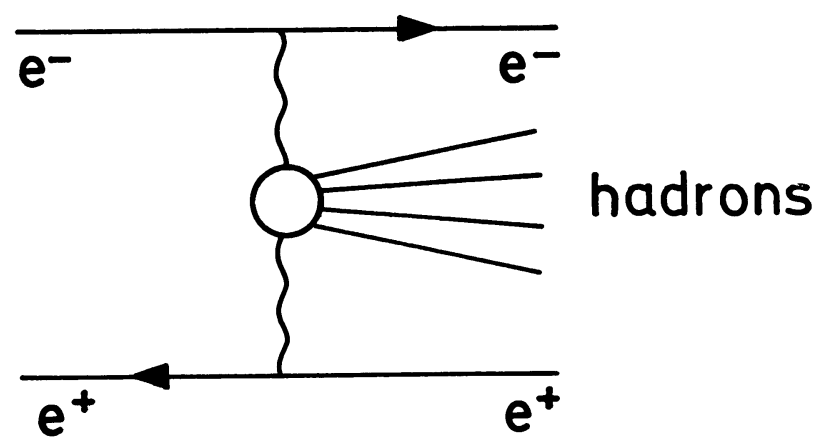

Fig. 1

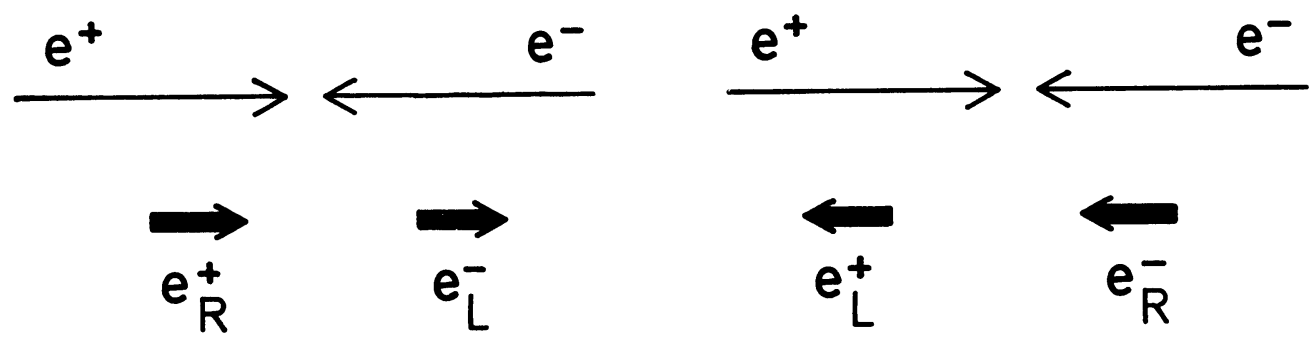

Fig. 2

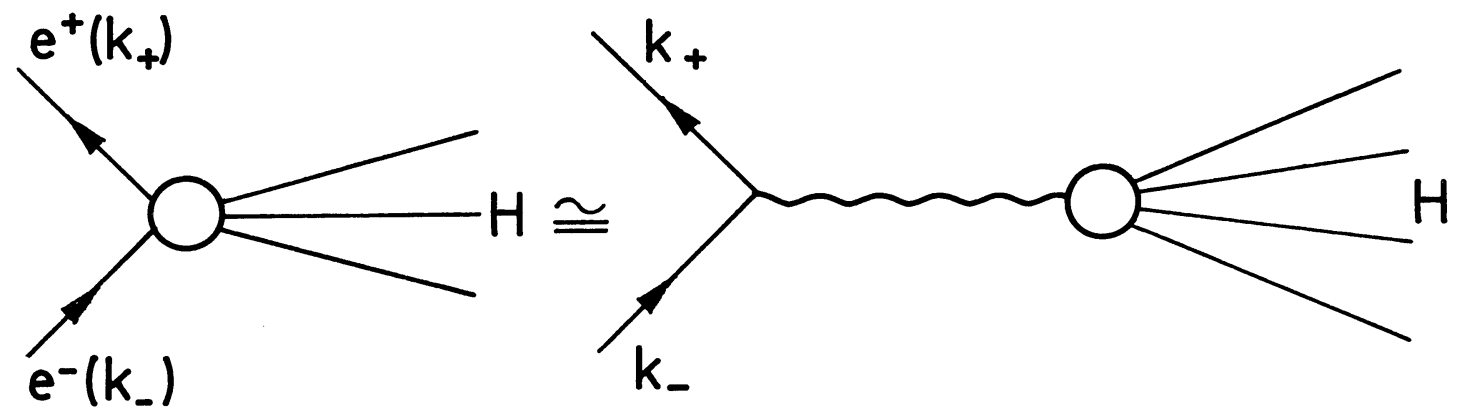

Fig. 3 


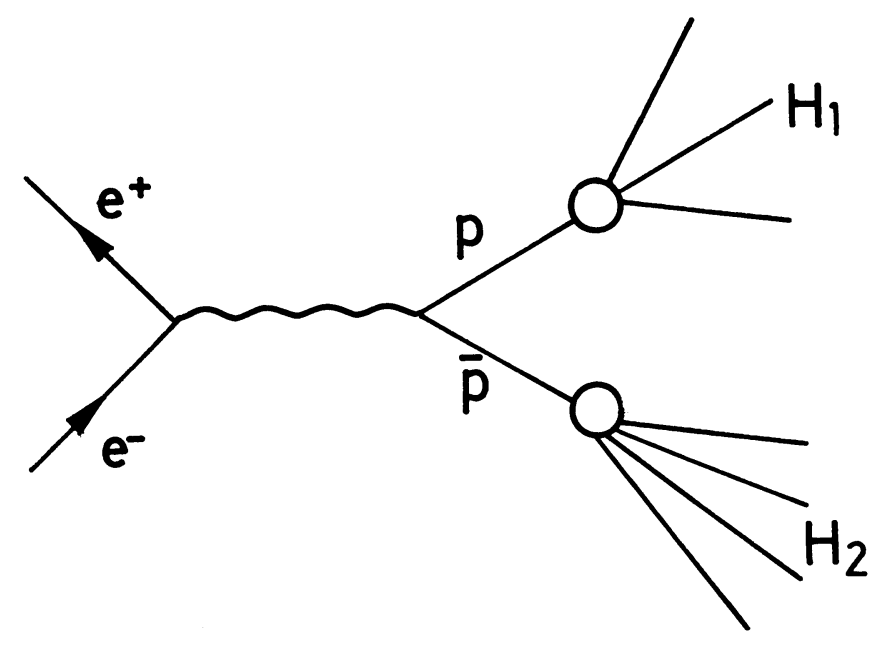

Fig. $4 a$

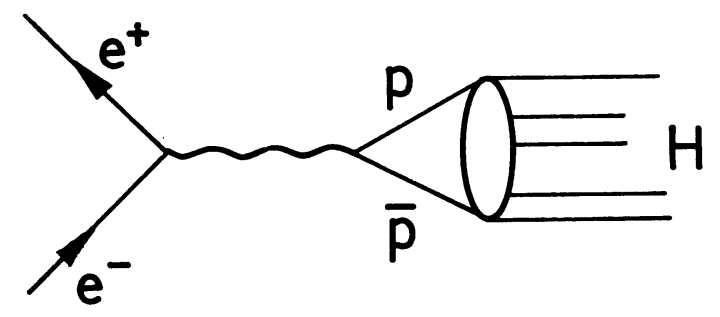

Fig. 4b 


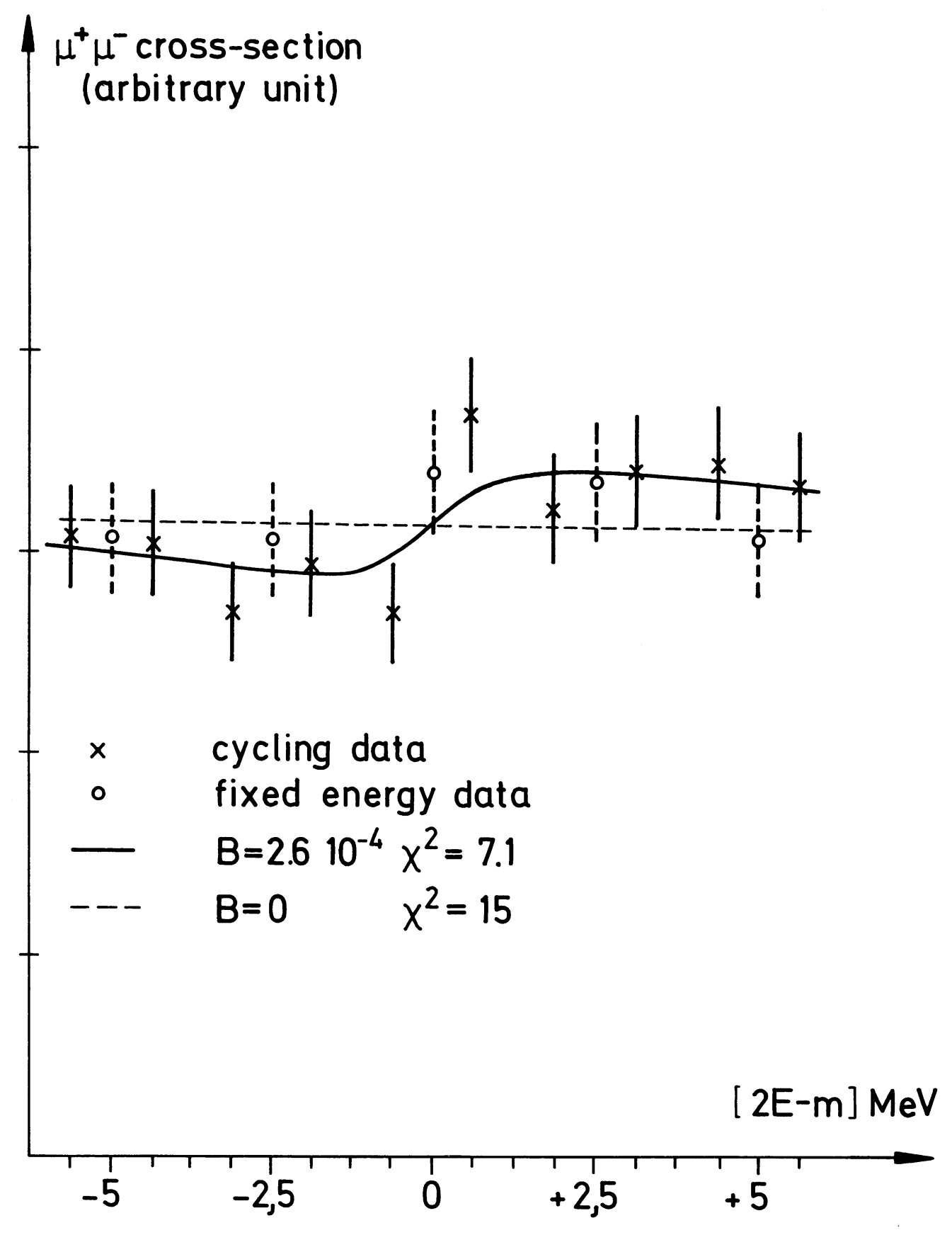

Fig. 5 


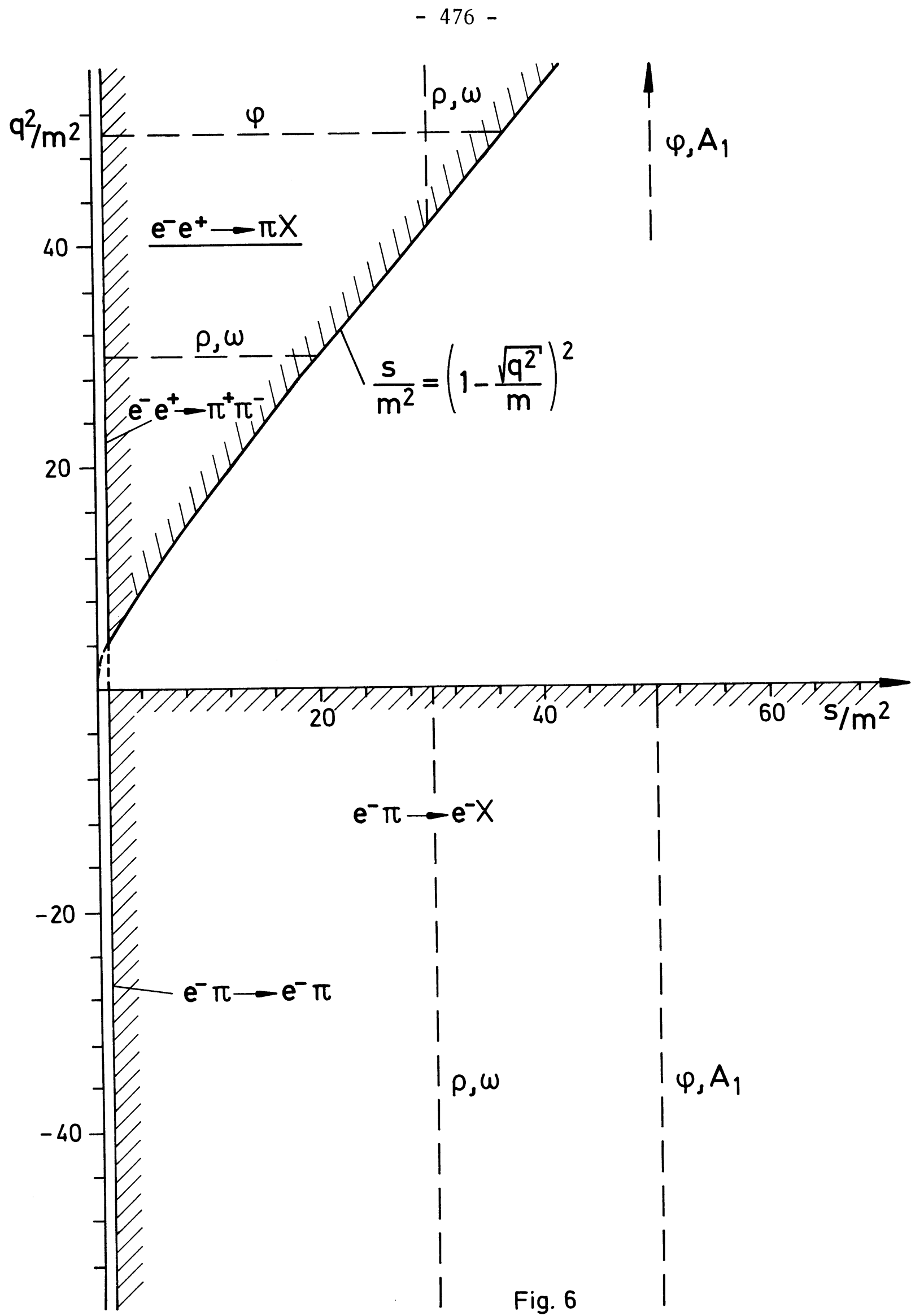

Fig. 6 


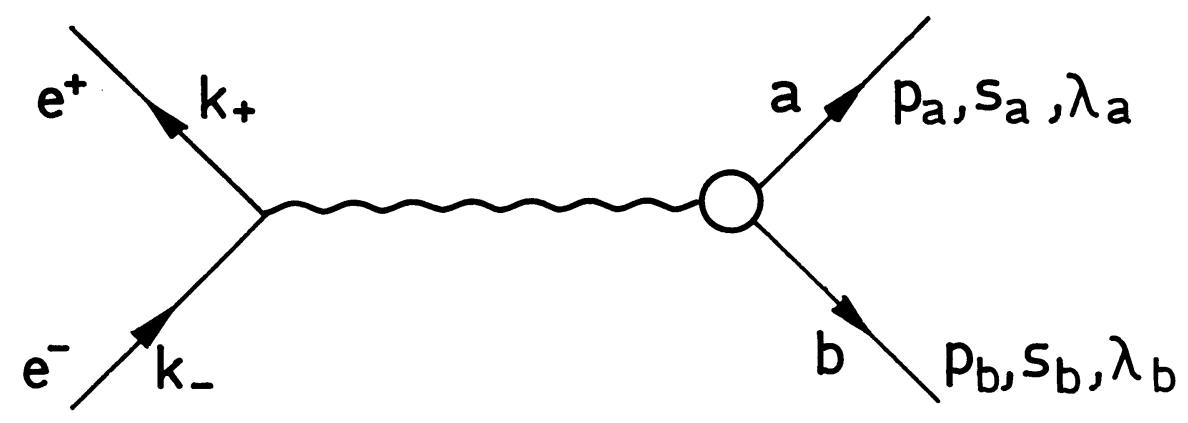

Fig. 7

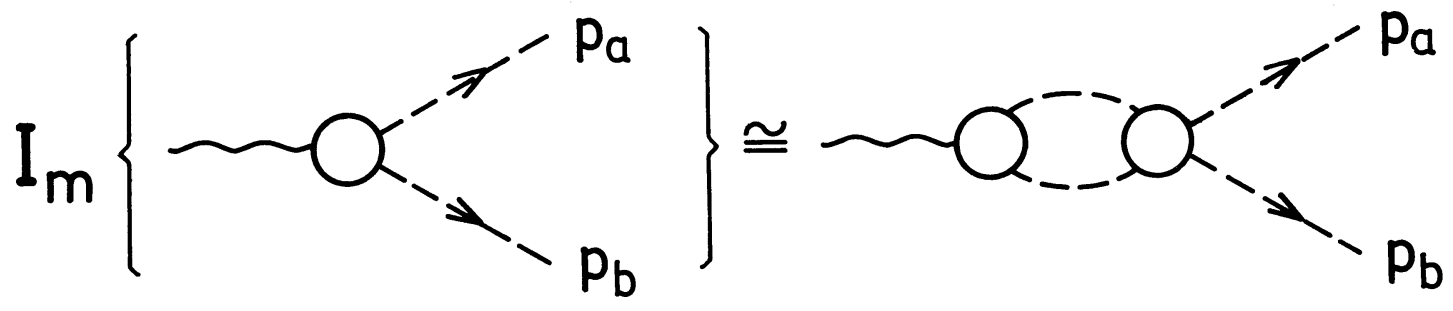

Fig. 8 


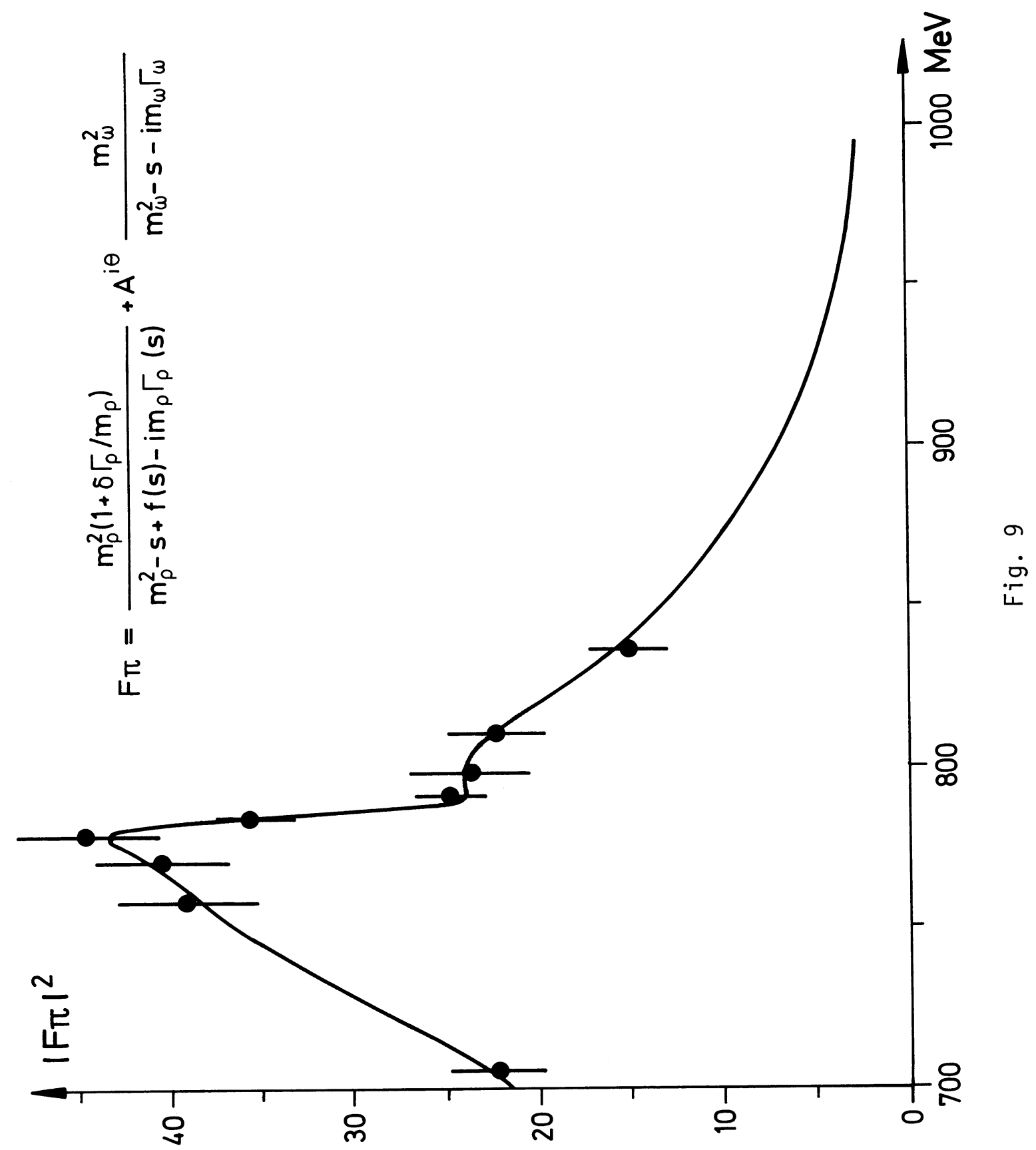




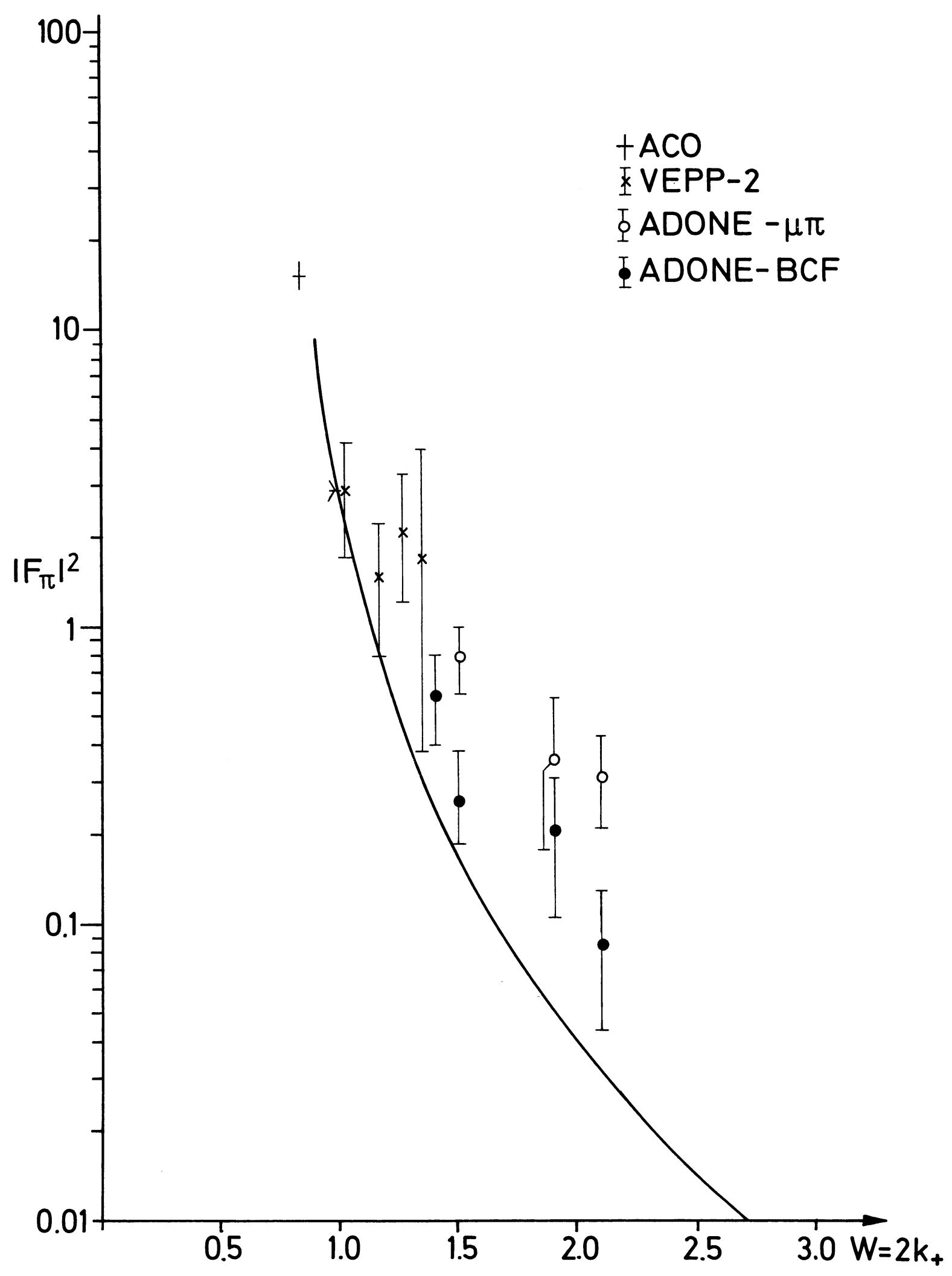

Fig. 10 
- 480 -

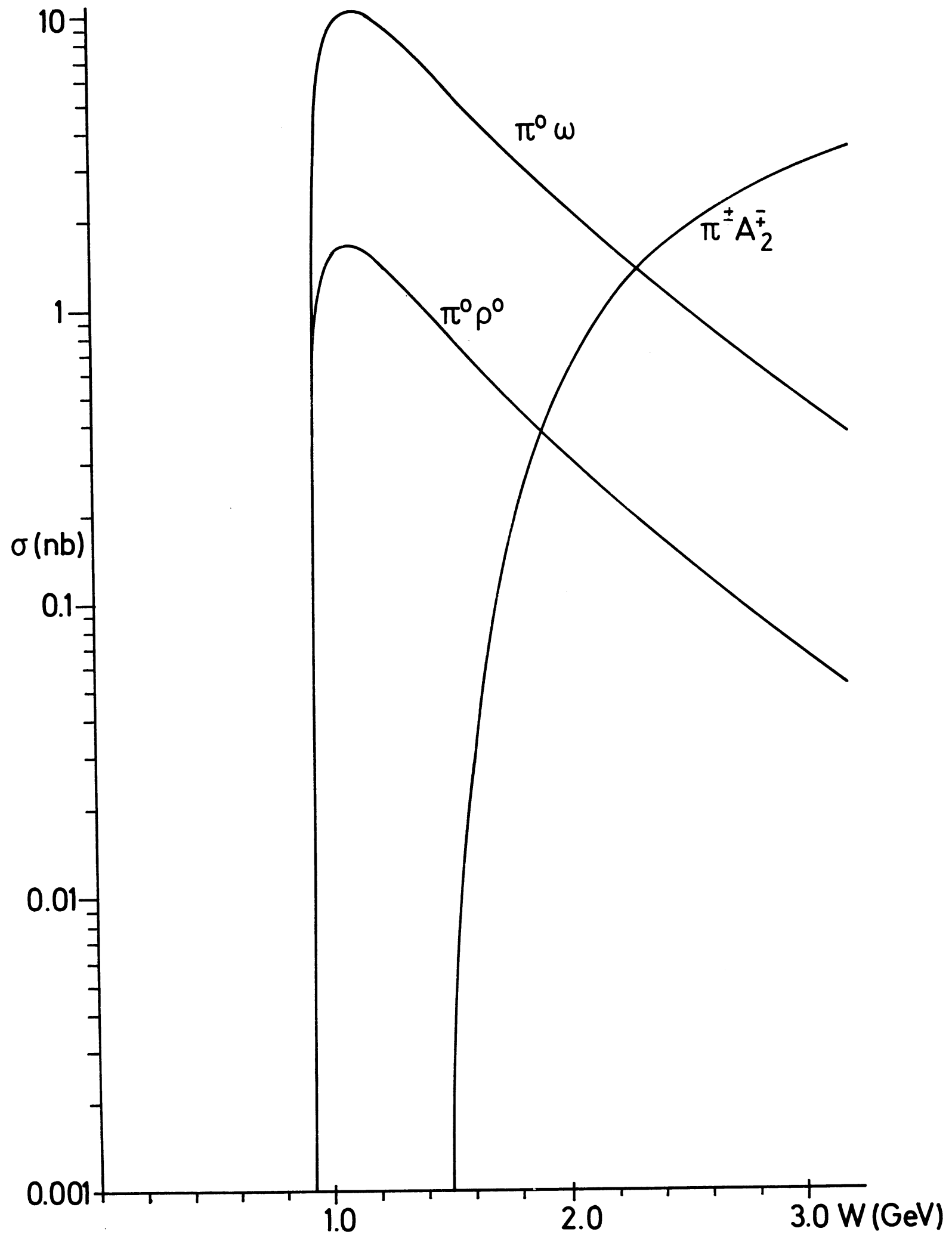

Fig. 11 


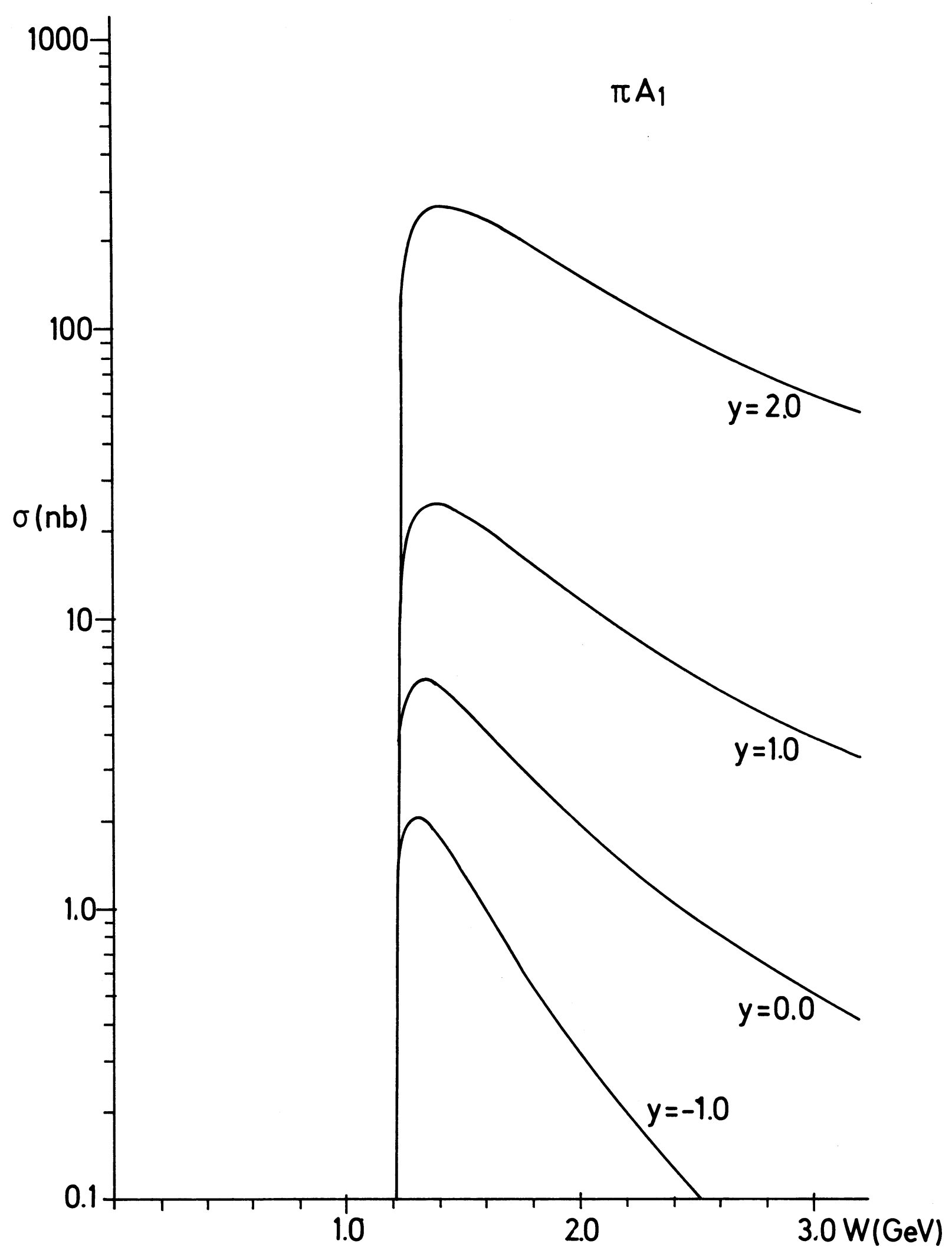

Fig. 12 


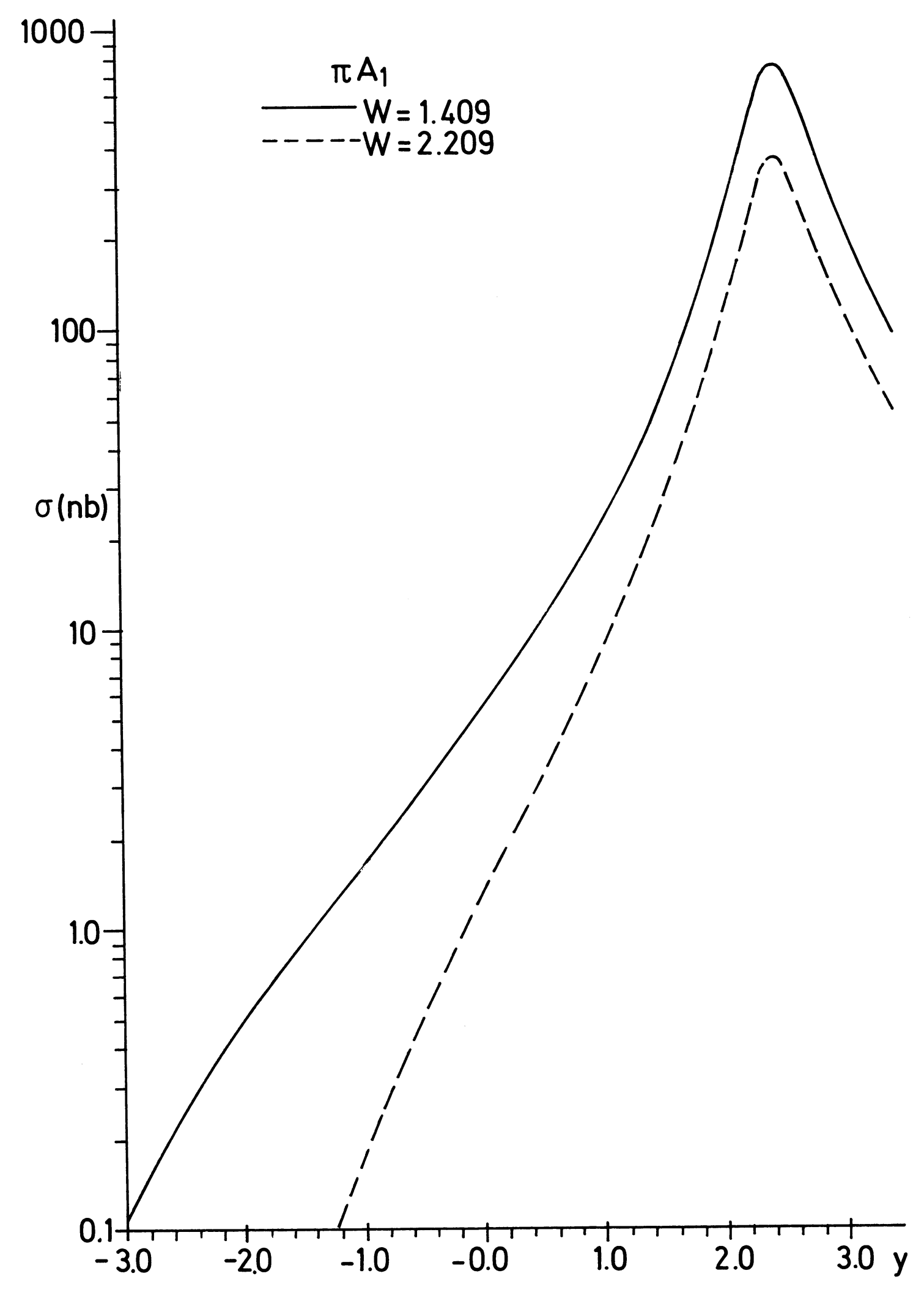

Fig. 13 


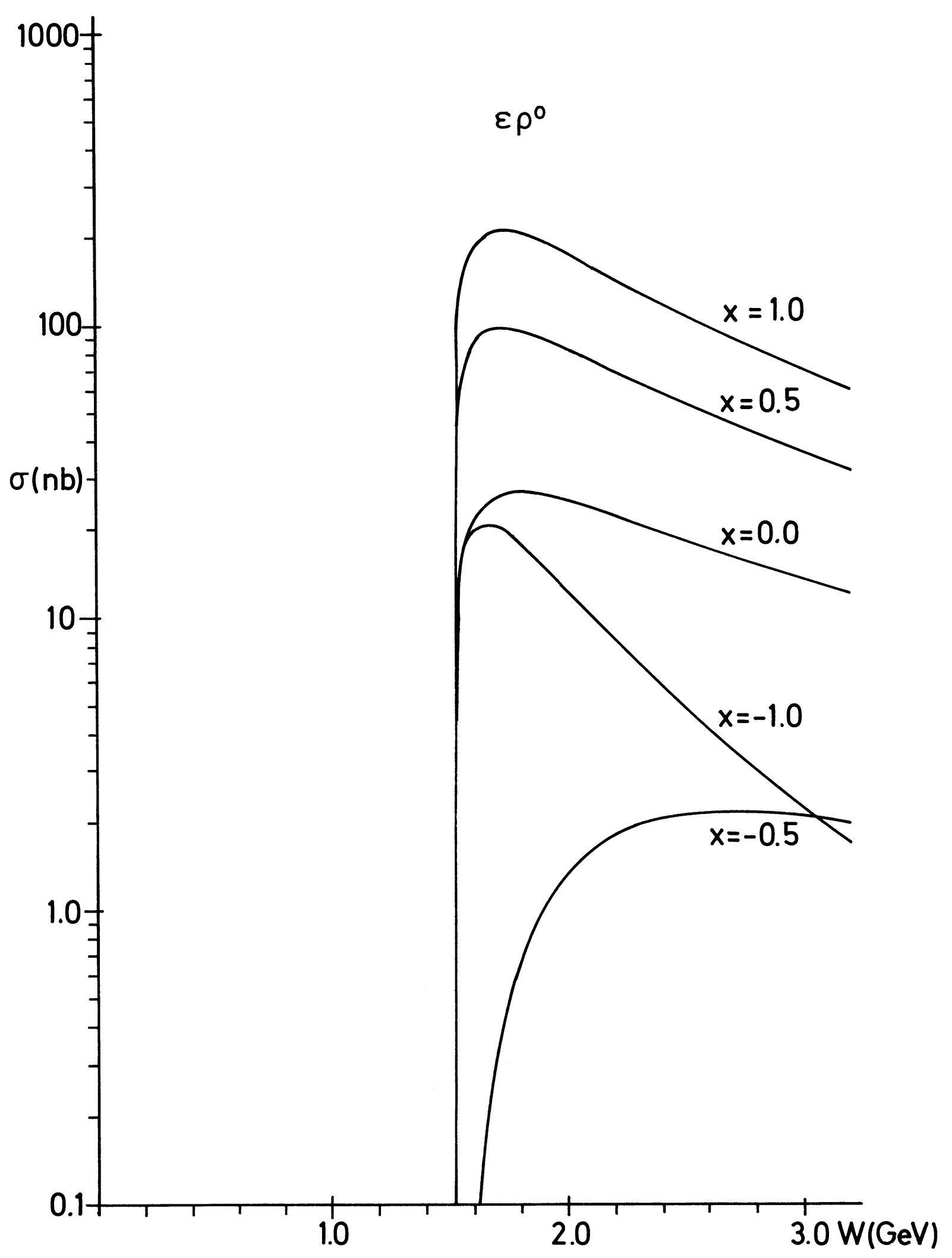

Fig. 14 

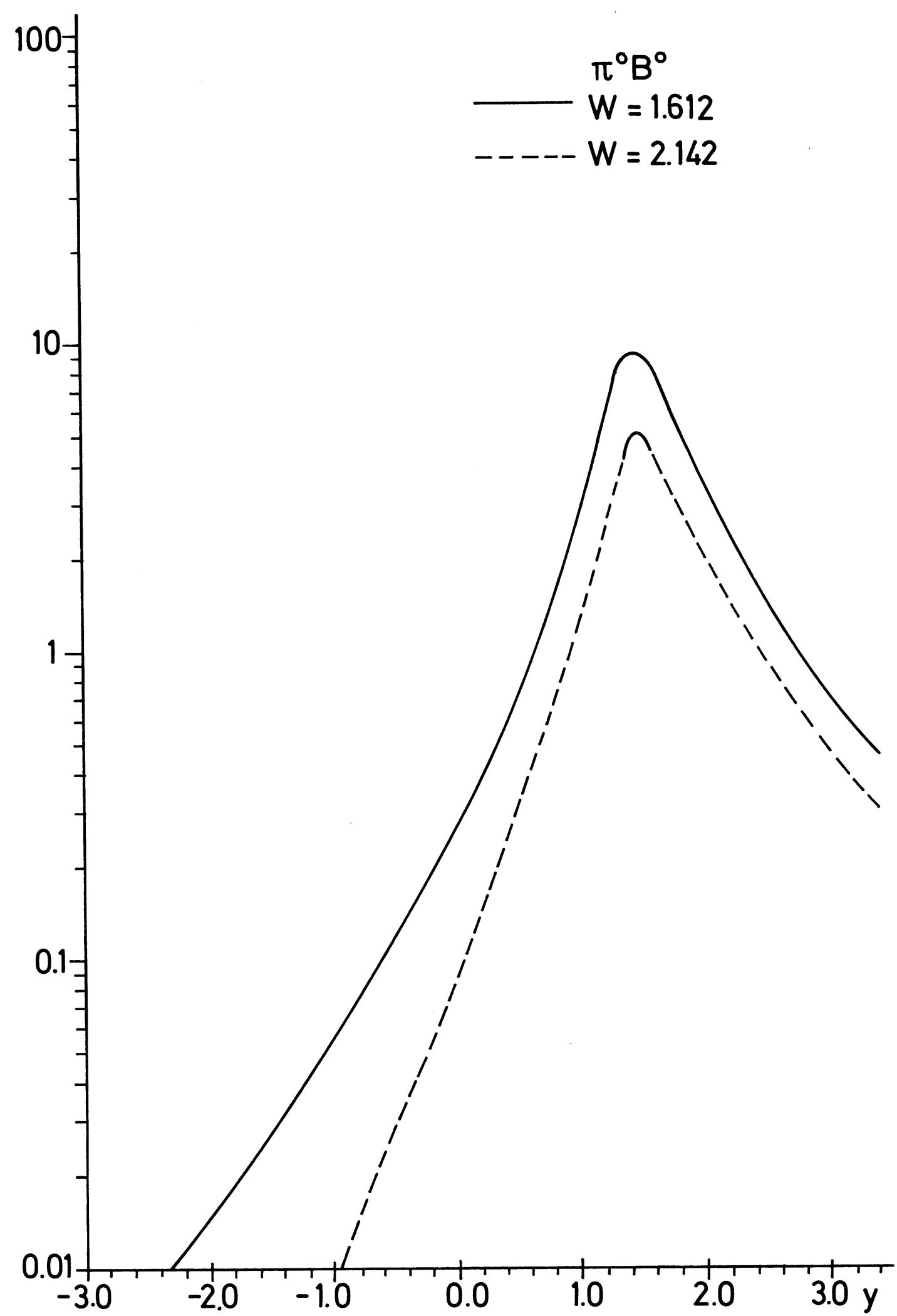

Fig. 15 


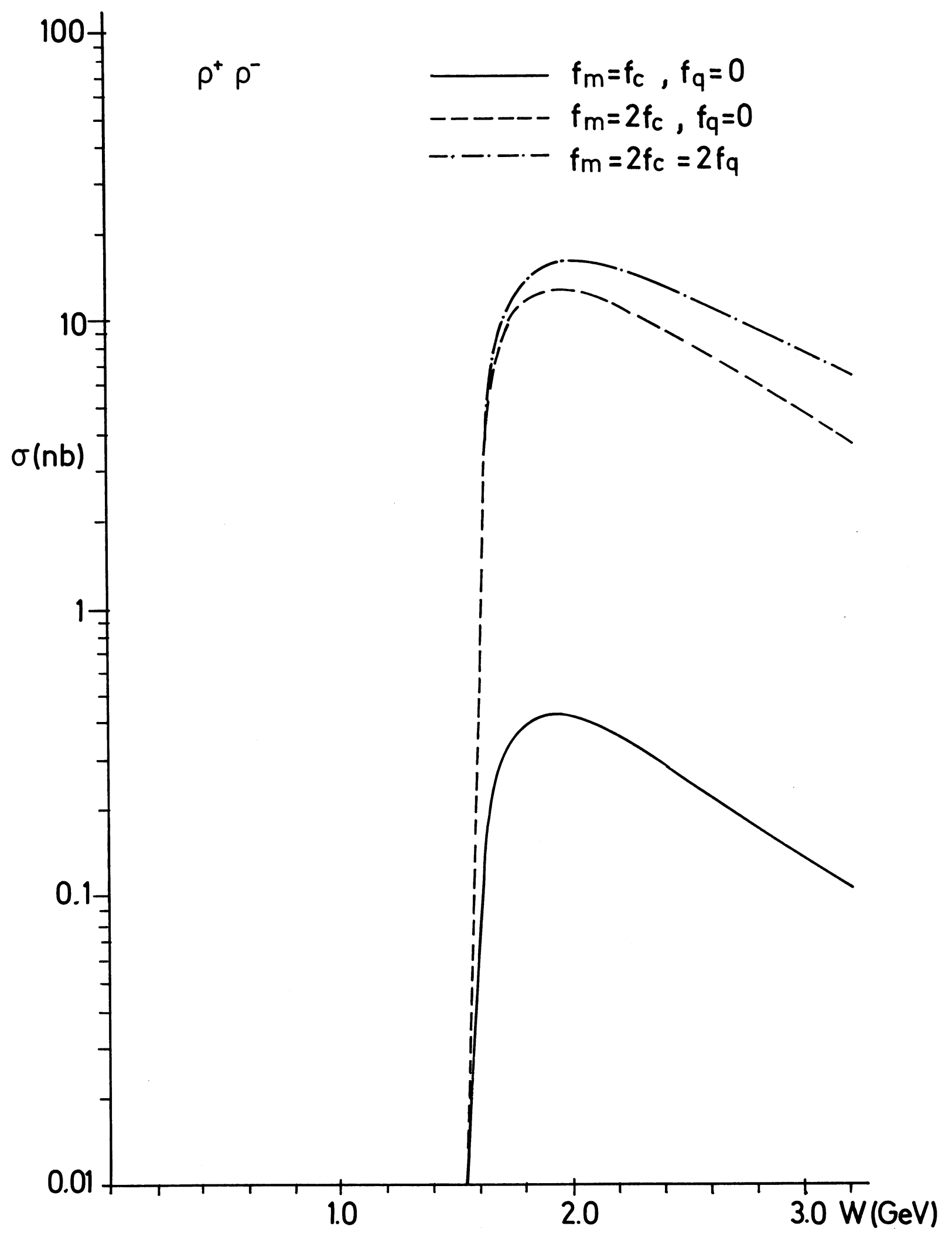

Fig. 16 


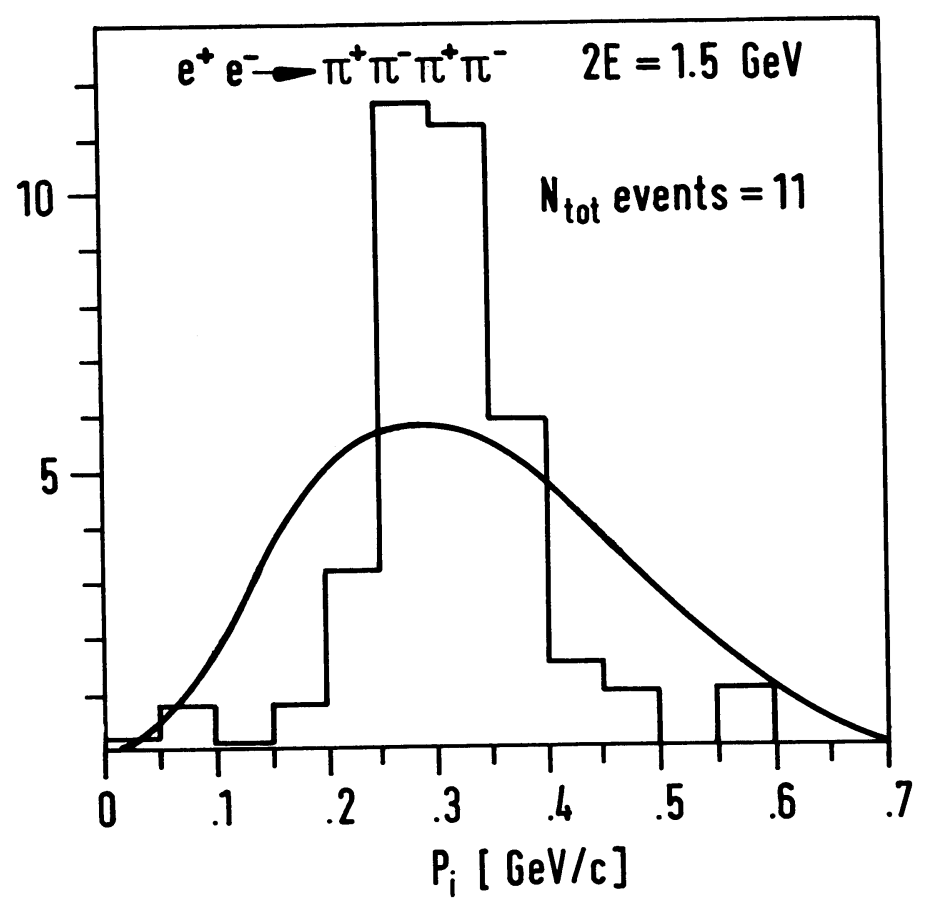

Fig. $17 a$

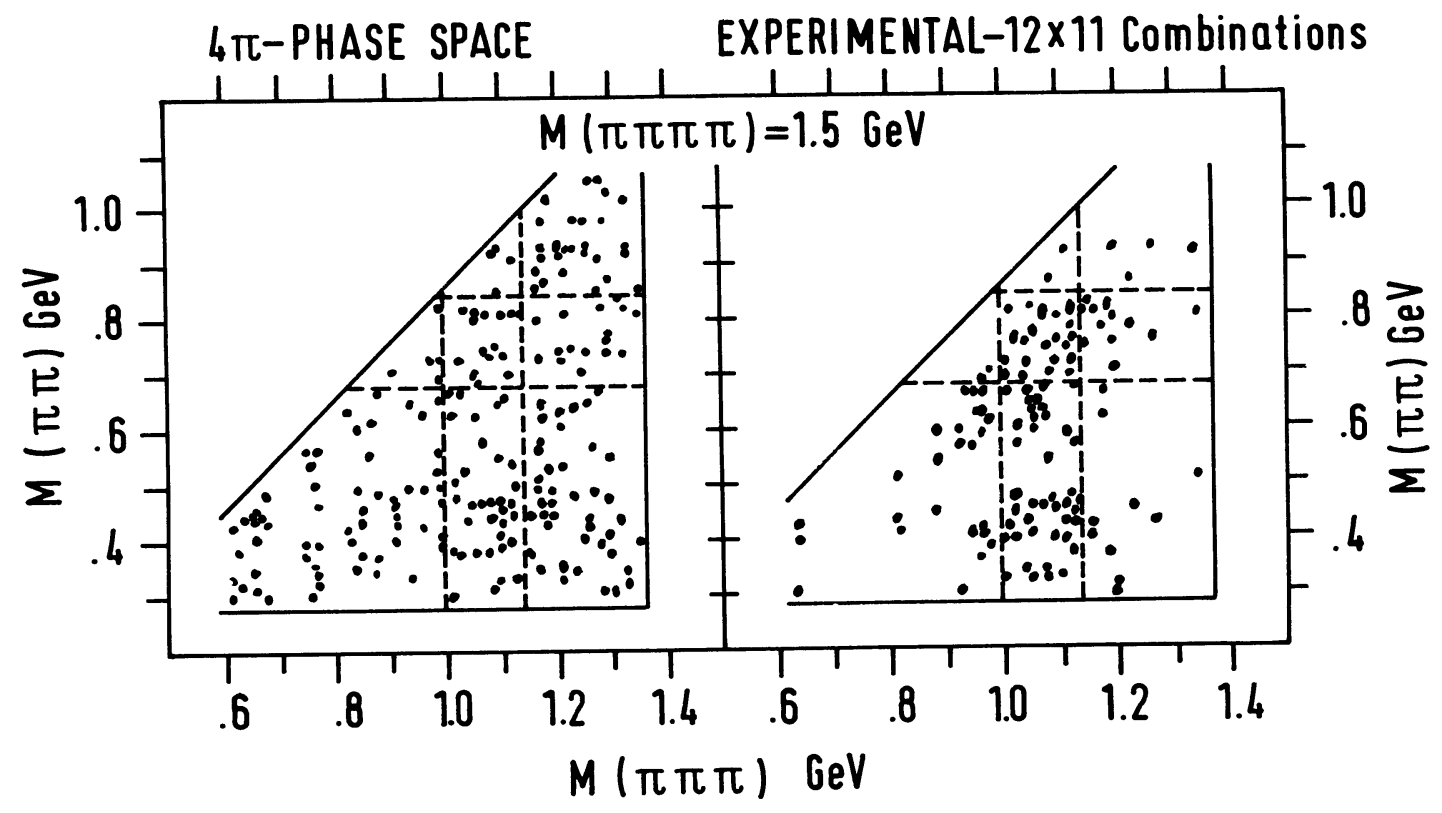

Fig. 17b 
$e^{+} e^{-}=\pi^{+} \pi^{-}+$Neutrals $\left(\sigma_{2}, n\right)$

$\otimes \quad \operatorname{ACO}\left(\mathrm{e}^{+} \mathrm{e}^{-}=\pi^{+} \pi^{-} \pi^{0} \pi^{0}\right)$

日 ADONE $\gamma \gamma\left(\pi^{+} \pi^{-}+n \pi^{0}, n=1 \div 4\right)$

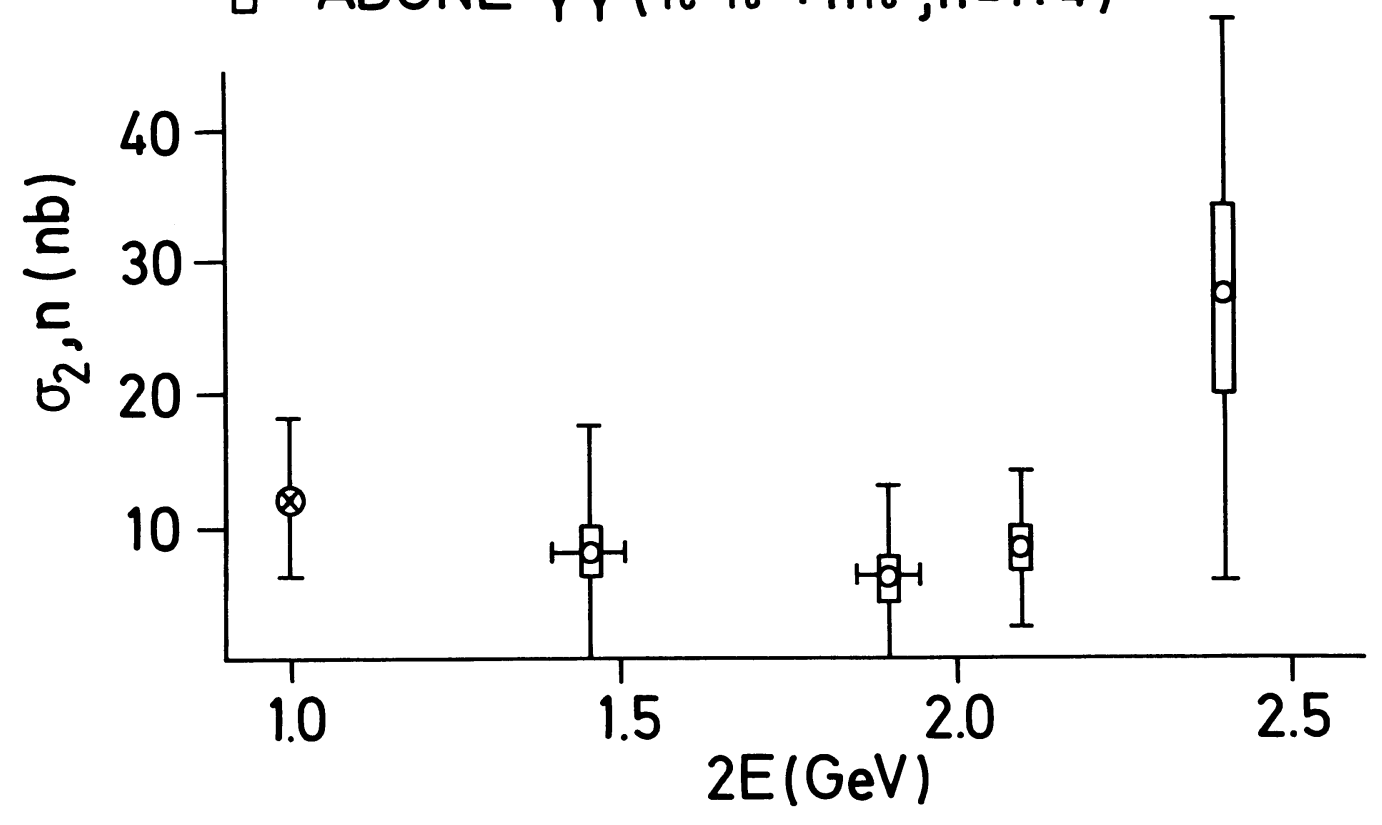

Fig. 18

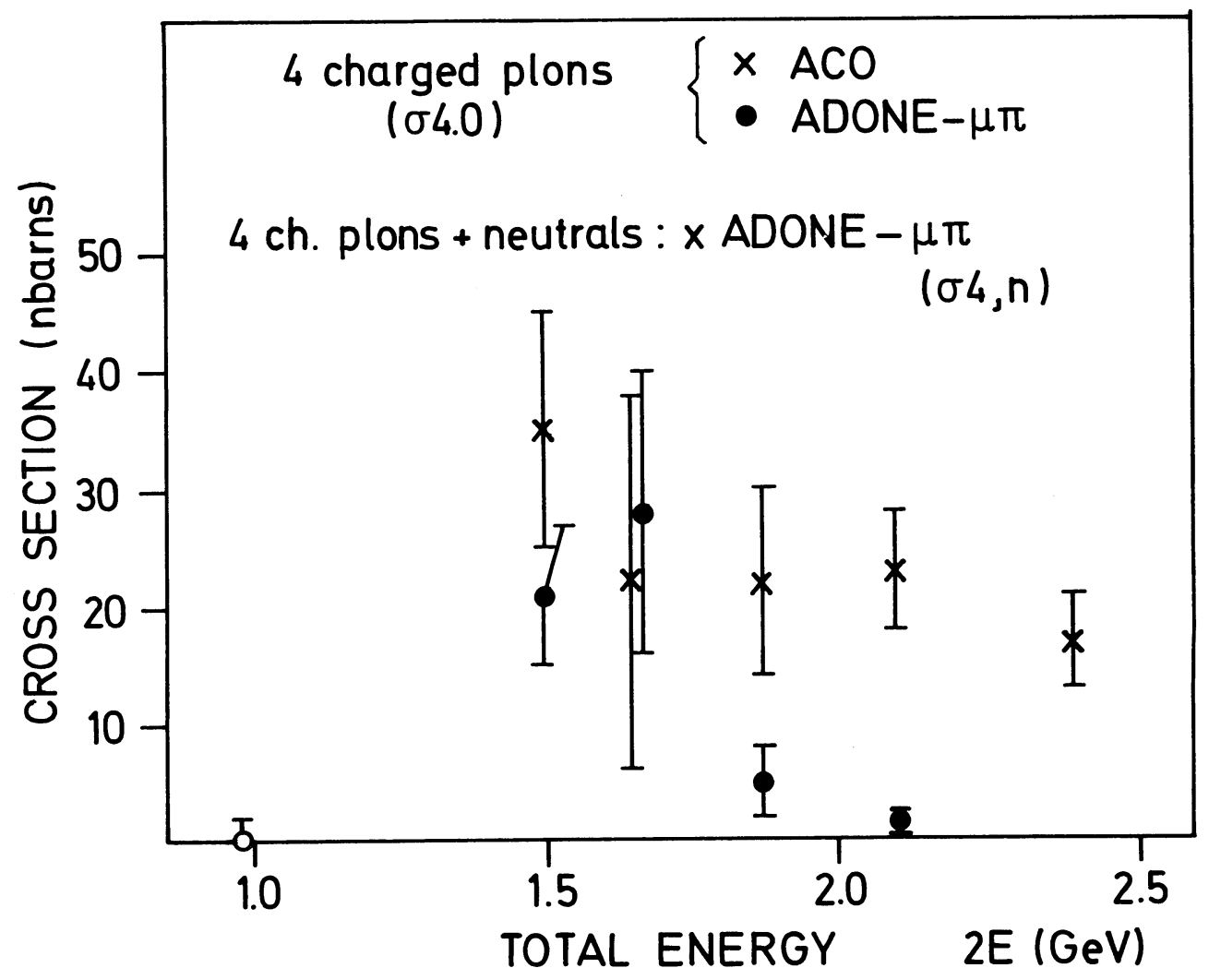

Fig. 19 
$e^{+} e^{-}-\pi^{+} \pi^{-} \pi^{+} \pi^{-}+$anything $(\sigma \geq 4)$

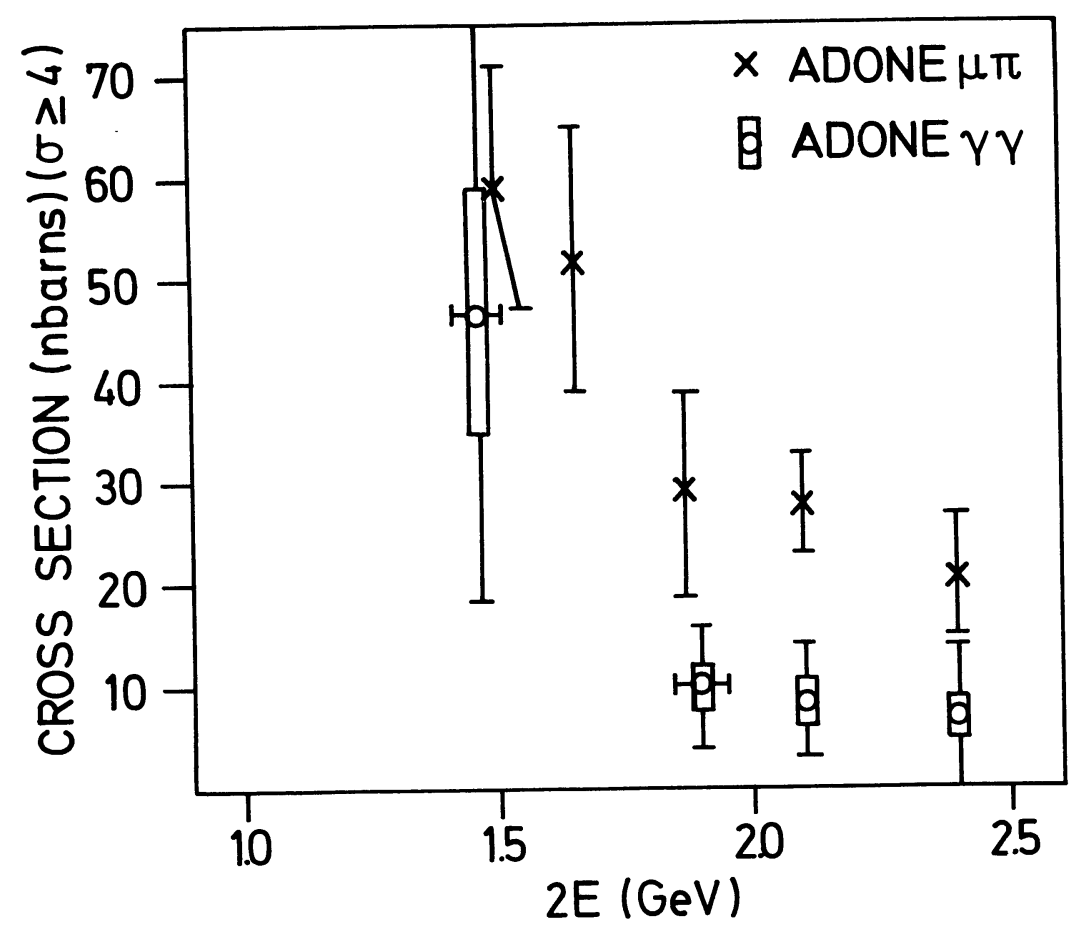

Fig. 20

$\mathrm{e}^{+} \mathrm{e}^{-}-$more than 2 charged pions

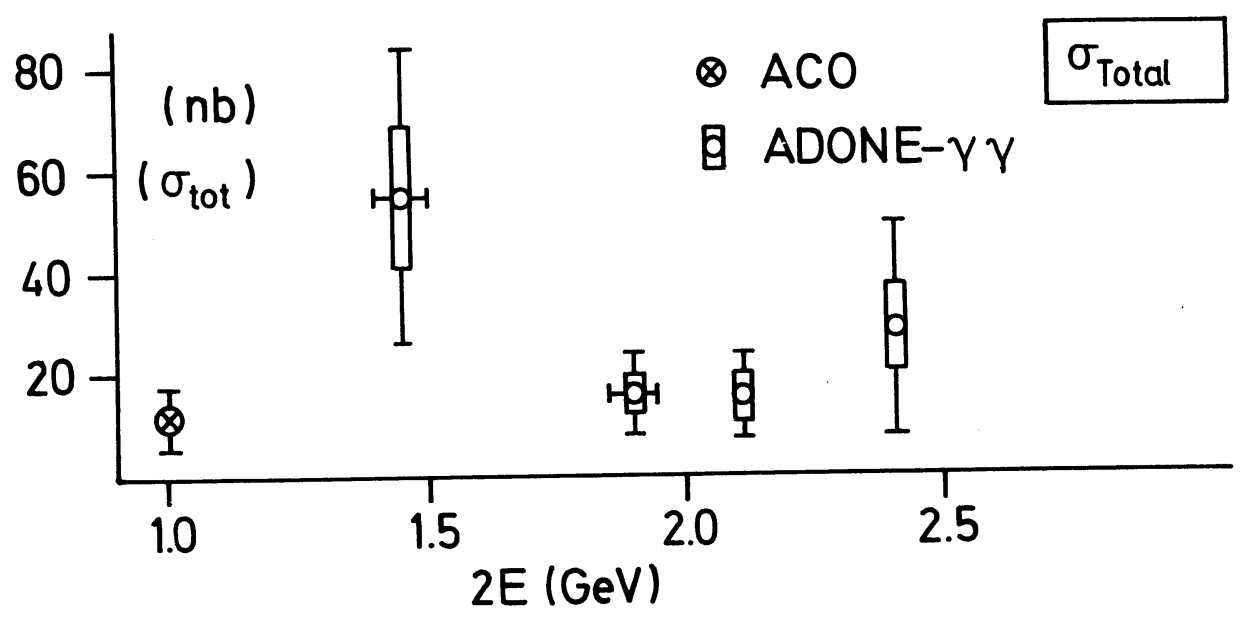

Fig. 21 

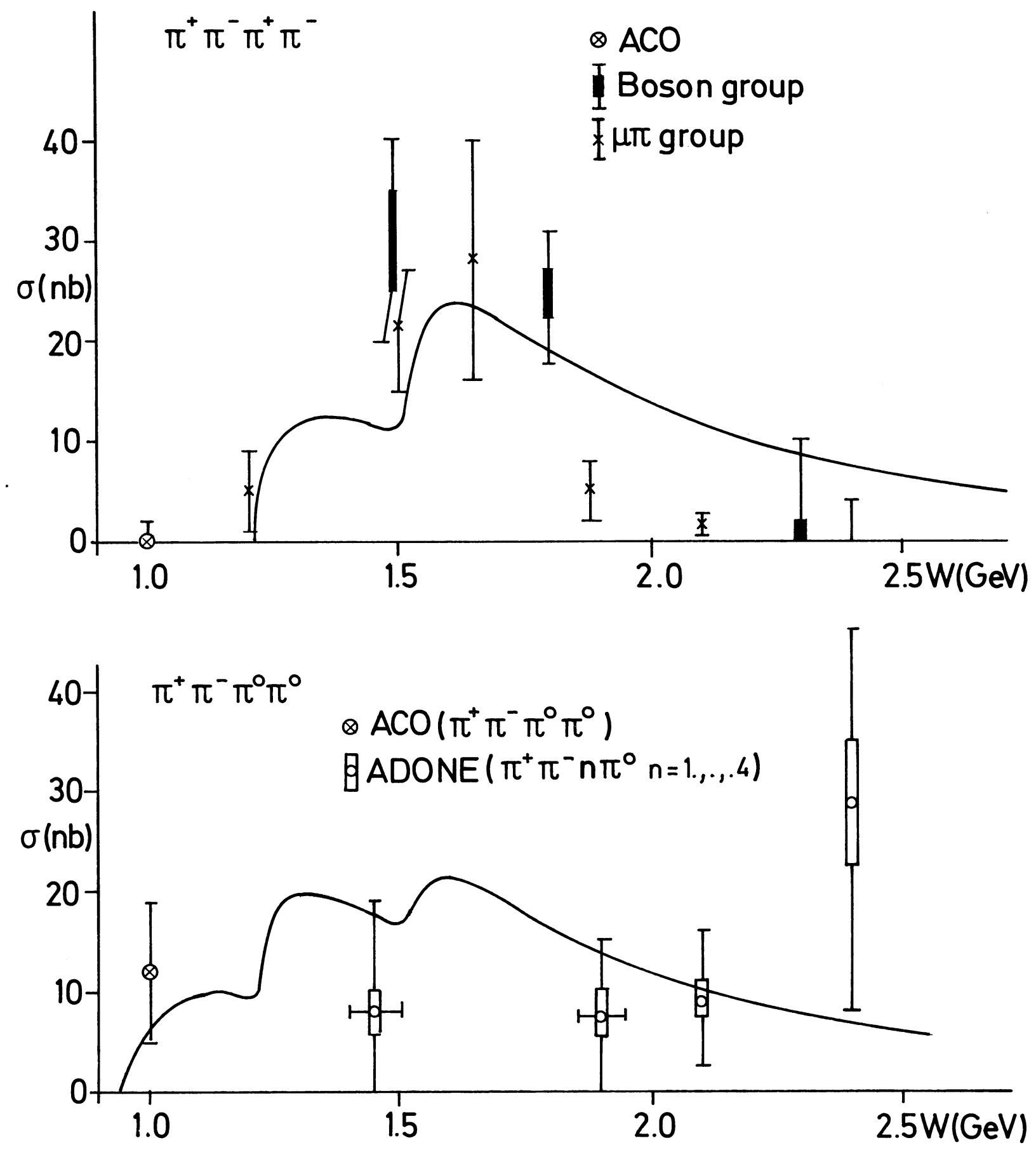

Fig. 22 


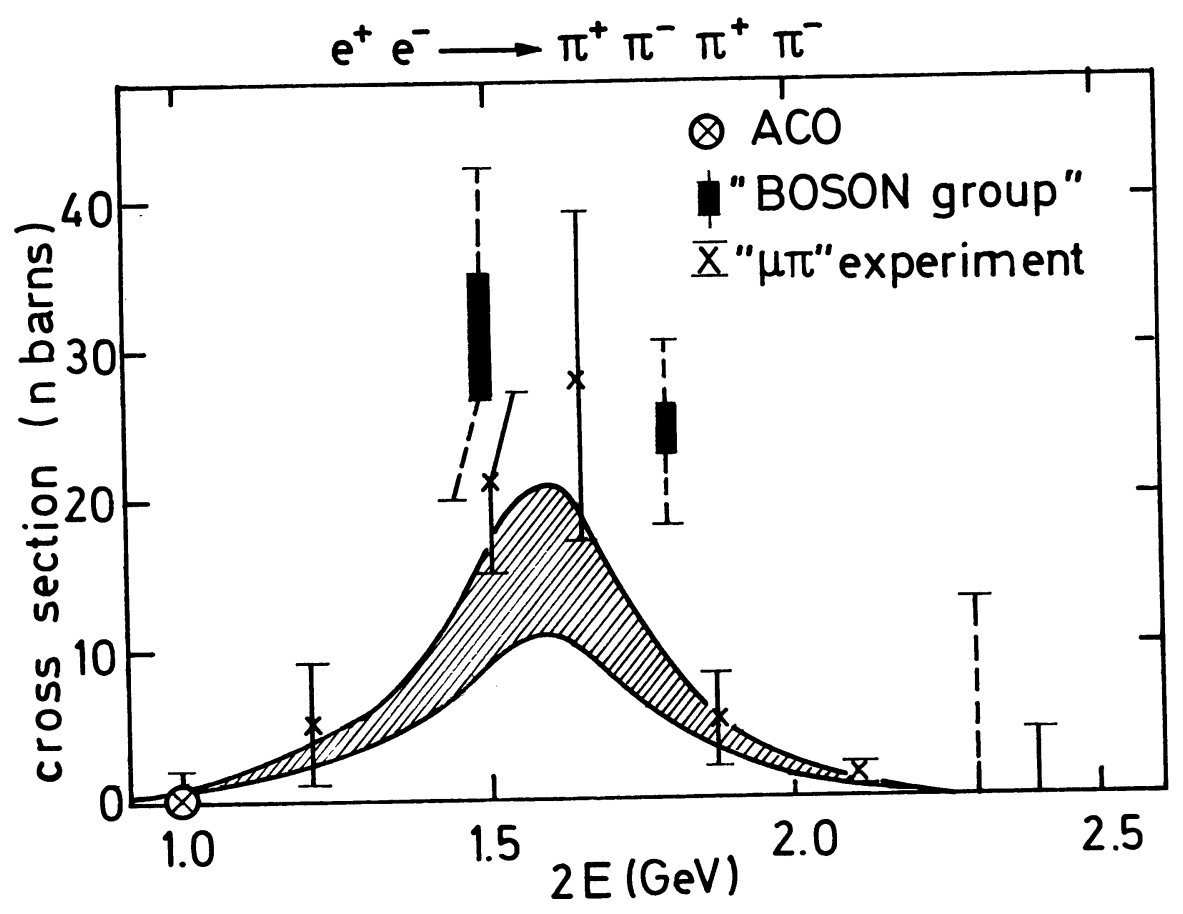

Fig. 23 